\title{
EXACT CONTROLLABILITY FOR PROBLEMS OF TRANSMISSION OF THE PLATE EQUATION WITH LOWER-ORDER TERMS
}

BY

\author{
WEIJIU LIU AND GRAHAM H. WILLIAMS
}

Dept. of Mathematics, Univ. of Wollongong, Northfields Avenue, Wollongong, NSW 2522, Australia

Abstract. We consider the exact controllability for the problem of transmission of the plate equation with lower-order terms. Using Lions' Hilbert Uniqueness Method (HUM for short), we show that the system is exactly controllable in $L^{2}(\Omega) \times H^{-2}(\Omega)$. We also obtain some uniqueness theorems for the problem of transmission of the plate equation and for the operator $a(x) \Delta^{2}+q$.

0. Introduction. This paper aims to consider the exact controllability for the problem of transmission of the plate equation with lower-order terms

$$
\begin{cases}y^{\prime \prime}+a(x) \Delta^{2} y+q y=0 & \text { in } Q, \\ y(0)=y^{0}, \quad y^{\prime}(0)=y^{1} & \text { in } \Omega, \\ y_{2}=0, \quad \frac{\partial y_{2}}{\partial \nu}=\phi & \text { on } \Sigma \\ y_{1}=y_{2}, \quad \frac{\partial y_{1}}{\partial \nu}=\frac{\partial y_{2}}{\partial \nu} & \text { on } \Sigma_{1} \\ a_{1} \Delta y_{1}=a_{2} \Delta y_{2}, \quad a_{1} \frac{\partial \Delta y_{1}}{\partial \nu}=a_{2} \frac{\partial \Delta y_{2}}{\partial \nu} & \text { on } \Sigma_{1} .\end{cases}
$$

In (0.1), $\Omega$ is a bounded domain in $\mathbb{R}^{n}(n \geq 1)$ with suitably smooth boundary $\Gamma=\partial \Omega$, and $\Omega_{1}$ is a bounded domain with $\bar{\Omega}_{1} \subset \Omega$ and suitably smooth boundary $\Gamma_{1}=\partial \Omega_{1}$; $\Omega_{2}=\Omega-\Omega_{1}, Q=\Omega \times(0, T), Q_{1}=\Omega_{1} \times(0, T), Q_{2}=\Omega_{2} \times(0, T), \Sigma=\Gamma \times(0, T)$, $\Sigma_{1}=\Gamma_{1} \times(0, T)$ for $T>0 ; \nu$ is the unit normal of $\Gamma$ or $\Gamma_{1}$ pointing towards the exterior of $\Omega$ or $\Omega_{1} ; y^{\prime}=\frac{\partial y}{\partial t}, y(0)=y(x, 0), y^{\prime}(0)=y^{\prime}(x, 0), y_{1}=\left.y\right|_{\Omega_{1}}, y_{2}=\left.y\right|_{\Omega_{2}} ; q(x, t)$ is a given function on $Q$ satisfying

$$
q \in L^{\infty}(Q)
$$

Received November 8, 1996.

2000 Mathematics Subject Classification. Primary 93B05, 35B37.

Key words and phrases. Exact controllability, plate equation, problems of transmission.

E-mail address: w.liu@uow.edu.au

E-mail address: ghw@uow.edu.au 
and $a(x)$ is given by

$$
a(x)= \begin{cases}a_{1}, & x \in \Omega_{1}, \\ a_{2}, & x \in \Omega_{2},\end{cases}
$$

where $a_{1}$ and $a_{2}$ are positive constants.

To be precise, the problem is: For suitable $T>0$ and every initial $\left(y^{0}, y^{1}\right)$ (given in a suitable Hilbert space), we want to find a corresponding control $\phi$ driving system (0.1) to rest at time $T$, that is, such that the solution $y(x, t ; \phi)$ of $(0.1)$ satisfies

$$
y(x, T ; \phi)=y^{\prime}(x, T ; \phi)=0 \quad \text { in } \Omega .
$$

Since the problem is linear, this is equivalent to steering the system to any state. This question was raised by Lions (see [10], p. 395) in the case of $q \equiv 0$.

There has been extensive work (see [1], [4]-[11]) over the past ten years on the problem of exact controllability for the plate equation. However, concerning the problem of transmission, there has been no study so far. In this paper, using Lions' Hilbert Uniqueness Method (HUM for short), we will show that under certain assumptions made on $\Omega_{1}, a(x)$, and $q(x)$ we can find a control $\phi \in L^{2}(\Sigma)$ steering system (0.1) from any initial state $\left(y^{0}, y^{1}\right) \in L^{2}(\Omega) \times H^{-2}(\Omega)$ to rest.

Meanwhile, we also obtain some uniqueness theorems for the problem of transmission of the plate equation and for the operator $a(x) \Delta^{2}+q$, which themselves are of significant importance. These theorems are in part the answer to the open question raised by Zuazua in $[14]$.

Throughout this paper, in addition to the above notation about $\Omega$ and $\Omega_{1}$, we also adopt the following notation. Let $x^{0} \in \mathbb{R}^{n}$ and set

$$
\begin{gathered}
m(x)=x-x^{0}=\left(x_{k}-x_{k}^{0}\right), \\
\Gamma\left(x^{0}\right)=\left\{x \in \Gamma: m(x) \cdot \nu(x)=m_{k}(x) \nu_{k}(x)>0\right\}, \\
\Gamma_{*}\left(x^{0}\right)=\Gamma-\Gamma\left(x^{0}\right)=\{x \in \Gamma: m(x) \cdot \nu(x) \leq 0\}, \\
\Sigma\left(x^{0}\right)=\Gamma\left(x^{0}\right) \times(0, T), \\
\Sigma_{*}\left(x^{0}\right)=\Gamma_{*}\left(x^{0}\right) \times(0, T), \\
R\left(x^{0}\right)=\max _{x \in \bar{\Omega}}|m(x)|=\max _{x \in \bar{\Omega}}\left|\sum_{k=1}^{n}\left(x_{k}-x_{k}^{0}\right)^{2}\right|^{\frac{1}{2}} .
\end{gathered}
$$

$H^{s}(\Omega)$ always denotes the usual Sobolev space and $\|\cdot\|_{s, \Omega}$ denotes its norm for any $s \in \mathbb{R}$. Let $X$ be a Banach space. We denote by $C^{k}([0, T], X)$ the space of all $k$ times continuously differentiable functions defined on $[0, T]$ with values in $X$, and write $C([0, T], X)$ for $C^{0}([0, T], X)$.

The plan for this paper is as follows. In Sec. 1, using the variational methods, we briefly discuss the well-posedness for the problem of transmission. Section 2 is devoted to the discussion of the boundary regularity of solutions. Section 3 concerns nonhomogeneous boundary value problems. In Sec. 4, the key estimates for the solutions (i.e., the socalled "observability inequality") are obtained. Finally, in Sec. 5, the theorems of exact controllability are given. 
1. Well-posedness. We shall use the variational methods to solve the problem

$$
\begin{cases}u^{\prime \prime}+a(x) \Delta^{2} u+q(x, t) u=f & \text { in } Q, \\ u(0)=u^{0}, \quad u^{\prime}(0)=u^{1} & \text { in } \Omega, \\ u_{2}=\frac{\partial u_{2}}{\partial \nu}=0, & \text { on } \Sigma, \\ u_{1}=u_{2}, \quad \frac{\partial u_{1}}{\partial \nu}=\frac{\partial u_{2}}{\partial \nu} & \text { on } \Sigma_{1}, \\ a_{1} \Delta u_{1}=a_{2} \Delta u_{2}, \quad a_{1} \frac{\partial \Delta u_{1}}{\partial \nu}=a_{2} \frac{\partial \Delta u_{2}}{\partial \nu} & \text { on } \Sigma_{1},\end{cases}
$$

where $u_{1}=\left.u\right|_{\Omega_{1}}$ and $u_{2}=\left.u\right|_{\Omega_{2}}$.

Set

$$
\begin{aligned}
H^{4}\left(\Omega, \Gamma_{1}\right)=\left\{u: u \in H_{0}^{2}(\Omega) ; u_{i} \in H^{4}\left(\Omega_{i}\right), i=1,2 ;\right. \\
\left.a_{1} \Delta u_{1}=a_{2} \Delta u_{1} \text { and } a_{1} \frac{\partial \Delta u_{1}}{\partial \nu}=a_{2} \frac{\partial \Delta u_{2}}{\partial \nu} \text { on } \Gamma_{1}\right\}
\end{aligned}
$$

with the norm

$$
\|u\|_{H^{4}\left(\Omega, \Gamma_{1}\right)}=\left(\left\|u_{1}\right\|_{4, \Omega_{1}}^{2}+\left\|u_{2}\right\|_{4, \Omega_{2}}^{2}\right)^{\frac{1}{2}} .
$$

It is well known that the norms $\|u\|_{4, \Omega}$ and $\left\|\Delta^{2} u\right\|_{0, \Omega}$ on $H^{4}(\Omega) \cap H_{0}^{2}(\Omega)$ are equivalent since $\Delta^{2}$ is an isomorphism from $H^{4}(\Omega) \cap H_{0}^{2}(\Omega)$ into $L^{2}(\Omega)$ (see [12], Vol. I, Chapter 2, p. 165). We generalize this result to the transmission case $H^{4}\left(\Omega, \Gamma_{1}\right)$. We first consider the regularity of the solution of

$$
\begin{cases}a(x) \Delta^{2} u=f & \text { in } \Omega, \\ u_{2}=\frac{\partial u_{2}}{\partial \nu}=0 & \text { on } \Gamma, \\ u_{2}=u_{2}, \quad \frac{\partial u_{1}}{\partial \nu}=\frac{\partial u_{2}}{\partial \nu} & \text { on } \Gamma_{1}, \\ a_{1} \Delta u_{1}=a_{2} \Delta u_{2}, \quad a_{1} \frac{\partial \Delta u_{1}}{\partial \nu}=a_{2} \frac{\partial \Delta u_{2}}{\partial \nu} & \text { on } \Gamma_{1} .\end{cases}
$$

LEMma 1.1. The solution of (1.4) belongs to $H^{4}\left(\Omega, \Gamma_{1}\right)$ for $f \in L^{2}(\Omega)$.

Proof. The solution $u$ of (1.4) can be written as

$$
u= \begin{cases}u_{1}, & x \in \Omega_{1}, \\ u_{2}, & x \in \Omega_{2},\end{cases}
$$

where $u_{1}, u_{2}$ are respectively the solutions of

$$
\begin{cases}a_{2} \Delta^{2} u_{2}=f & \text { in } \Omega, \\ u_{2}=\frac{\partial u_{2}}{\partial \nu}=0 & \text { on } \Gamma\end{cases}
$$

and

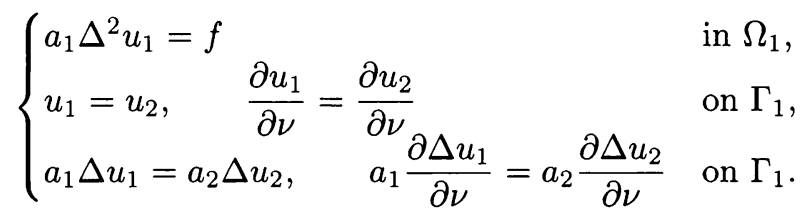


It follows from the elliptic regularity that $u_{2} \in H^{4}(\Omega)$. By the trace theorem we deduce that $u_{2} \in H^{\frac{7}{2}}\left(\Gamma_{1}\right)$ and $\frac{\partial u_{2}}{\partial \nu} \in H^{\frac{5}{2}}\left(\Gamma_{1}\right)$. Again by the elliptic regularity we have $u_{1} \in$ $H^{4}\left(\Omega_{1}\right)$. Thus $u \in H^{4}\left(\Omega, \Gamma_{1}\right)$.

Lemma 1.2. Suppose the boundaries $\Gamma$ and $\Gamma_{1}$ of $\Omega$ and $\Omega_{1}$ are of class $C^{4}$. Then the norm $\|u\|_{H^{4}\left(\Omega . \Gamma_{1}\right)}$ on $H^{4}\left(\Omega, \Gamma_{1}\right)$ defined by (1.3) is equivalent to

$$
|u|_{H^{4}\left(\Omega 2 . \Gamma_{1}\right)}=\left(\left\|\Delta^{2} u_{1}\right\|_{0 . \Omega_{1}}^{2}+\left\|\Delta^{2} u_{2}\right\|_{0, \Omega_{2}}^{2}\right)^{\frac{1}{2}} .
$$

Proof. The sesquilinear form $\alpha(u, v)$ associated with problem (1.4) is

$$
\alpha(u, v)=\int_{\Omega} a(x) \Delta u \Delta v d x, \quad \forall u, v \in H_{0}^{2}(\Omega) .
$$

It is easy to see that the sesquilinear form $\alpha$ is coercive on $H_{0}^{2}(\Omega)$. Let $A$ be the operator associated with the sesquilinear form $\alpha$ by

$$
\alpha(u, v)=\langle A u, v\rangle, \quad \forall u, v \in H_{0}^{2}(\Omega),
$$

where $\langle\cdot, \cdot\rangle$ denotes the dual product between the spaces $H_{0}^{2}(\Omega)$ and $H^{-2}(\Omega)$. By the Lax-Milgram theorem, $A$ is an isomorphism of $H_{0}^{2}(\Omega)$ onto $H^{-2}(\Omega)$. Set

$$
D(A)=\left\{u \in H_{0}^{2}(\Omega): A u \in L^{2}(\Omega)\right\} .
$$

Then by Lemma 1.1 we have

$$
D(A)=H^{4}\left(\Omega, \Gamma_{1}\right) .
$$

By Proposition 9 of $[2$, p. 370$], H^{4}\left(\Omega, \Gamma_{1}\right)$ provided with the norm of the graph

$$
\|u\|_{D(A)}=\left(\|u\|_{L^{2}(\Omega)}^{2}+\|A u\|_{L^{2}(\Omega)}^{2}\right)^{\frac{1}{2}}
$$

is a Banach space. In addition, $H^{4}\left(\Omega, \Gamma_{1}\right)$ with the norm $\|\cdot\|_{H^{4}\left(\Omega, \Gamma_{1}\right)}$ is also a Banach space, and the norm $\|\cdot\|_{H^{4}\left(\Omega . \Gamma_{1}\right)}$ is stronger than $\|\cdot\|_{D(A)}$. By the Banach open mapping theorem, these two norms $\|u\|_{H^{4}\left(\Omega, \Gamma_{1}\right)}$ and $\|u\|_{D(A)}$ on $H^{4}\left(\Omega, \Gamma_{1}\right)$ are equivalent. Again by the Banach open mapping theorem, $A$ is an isomorphism of $H^{4}\left(\Omega, \Gamma_{1}\right)$ onto $L^{2}(\Omega)$ with $H^{4}\left(\Omega, \Gamma_{1}\right)$ provided with the norm of the graph since $A: H^{4}\left(\Omega, \Gamma_{1}\right) \rightarrow L^{2}(\Omega)$ is continuous, one-to-one, and onto. Consequently, $A$ is also an isomorphism of $H^{4}\left(\Omega, \Gamma_{1}\right)$ onto $L^{2}(\Omega)$ with $H^{4}\left(\Omega, \Gamma_{1}\right)$ provided with the norm $\|\cdot\|_{H^{4}\left(\Omega, \Gamma_{1}\right)}$. It therefore follows that the norm $\|u\|_{H^{4}\left(\Omega, \Gamma_{1}\right)}$ is equivalent to $|u|_{H^{4}\left(\Omega, \Gamma_{1}\right)}$ on $H^{4}\left(\Omega, \Gamma_{1}\right)$ since $A=a(x) \Delta^{2}$ on $H^{4}\left(\Omega, \Gamma_{1}\right)$.

LEMma 1.3. $H^{4}\left(\Omega, \Gamma_{1}\right)$ is dense in $H_{0}^{2}(\Omega)$.

Proof. Let $H_{0}^{2}(\Omega)$ be provided with the following scalar product:

$$
\langle u, v\rangle=\int_{\Omega} a(x) \Delta u \Delta v d x, \quad \forall u, v \in H_{0}^{2}(\Omega),
$$

which is equivalent to the usual one.

Let $w \in H_{0}^{2}(\Omega)$ be such that

$$
\langle u, w\rangle=0, \quad \forall u \in H^{4}\left(\Omega, \Gamma_{1}\right) .
$$


Then

$$
0=\int_{\Omega} a(x) \Delta u \Delta w d x=\int_{\Omega} a(x) w \Delta^{2} u d x .
$$

By Lemma 1.1, $a(x) \Delta^{2} u$ runs over all $L^{2}(\Omega)$ when $u$ runs over all $H^{4}\left(\Omega, \Gamma_{1}\right)$. Thus $w=0$. It therefore follows from the Hahn-Banach theorem that $H^{4}\left(\Omega, \Gamma_{1}\right)$ is dense in $H_{0}^{2}(\Omega)$.

Lemma 1.3 is not obvious since $C_{0}^{\infty}(\Omega) \not \subset H^{4}\left(\Omega, \Gamma_{1}\right)$.

Let $\lambda_{1}$ be the smallest eigenvalue of the operator $\Delta^{2}$ with Dirichlet homogeneous boundary conditions on $L^{2}(\Omega)$, that is,

$$
\begin{cases}\Delta^{2} u=\lambda_{1} u & \text { in } \Omega \\ u=\frac{\partial u}{\partial \nu}=0 & \text { on } \Gamma .\end{cases}
$$

Then

$$
\|u\|_{0} \leq \frac{1}{\sqrt{\lambda_{1}}}\|\Delta u\|_{0}, \quad \forall u \in H_{0}^{2}(\Omega) .
$$

Since we have for $u \in H^{2}(\Omega) \cap H_{0}^{1}(\Omega)$

$$
\int_{\Omega}|\nabla u|^{2} d x=\int_{\Omega}-u \Delta u d x \leq\|u\|_{0}\|\Delta u\|_{0}
$$

we deduce from (1.7) that

$$
\|\nabla u\|_{0} \leq \frac{1}{\lambda_{1}^{1 / 4}}\|\Delta u\|_{0}, \quad \forall u \in H_{0}^{2}(\Omega) .
$$

We define the energy of system (1.1) by

$$
E(u, t)=\frac{1}{2} \int_{\Omega}\left[\left|u^{\prime}(x, t)\right|^{2}+a(x)|\Delta u|^{2}\right] d x .
$$

TheOREm 1.4. (i) Suppose that the boundaries $\Gamma$ and $\Gamma_{1}$ are of class $C^{2}$. Then for every initial condition $\left(u^{0}, u^{1}\right) \in H_{0}^{2}(\Omega) \times L^{2}(\Omega)$ and $f \in L^{1}\left(0, T ; L^{2}(\Omega)\right)$, problem (1.1) has a unique weak solution with

$$
u(t) \in C\left([0, T] ; H_{0}^{2}(\Omega)\right) \cap C^{1}\left([0, T] ; L^{2}(\Omega)\right) .
$$

Moreover, there exists a constant $c>0$ such that for all $t \in[0, T]$

$$
\|u(t)\|_{2, \Omega}+\left\|u^{\prime}(t)\right\|_{0, \Omega} \leq c\left[\left\|u^{0}\right\|_{2, \Omega}+\left\|u^{1}\right\|_{0, \Omega}+\|f\|_{L^{1}\left(0, T ; L^{2}(\Omega)\right.}\right] .
$$

(ii) Suppose that the boundary $\Gamma$ of $\Omega$ and the boundary $\Gamma_{1}$ of $\Omega_{1}$ are of class $C^{4}$. Assume that $q \in L^{\infty}\left(0, T ; W^{2, \infty}(\Omega)\right)$. Then for any initial condition $\left(u^{0}, u^{1}\right) \in$ $H^{4}\left(\Omega, \Gamma_{1}\right) \times H_{0}^{2}(\Omega)$ and $f \in L^{1}\left(0, T ; H_{0}^{2}(\Omega)\right)$, the problem (1.1) has a unique strong solution with

$$
u(t) \in C\left([0, T] ; H^{4}\left(\Omega, \Gamma_{1}\right)\right) \cap C^{1}\left([0, T] ; H_{0}^{2}(\Omega)\right) .
$$

Moreover, there exists a constant $c>0$ such that for all $t \in[0, T]$

$$
\begin{aligned}
& \left\|u^{\prime}(t)\right\|_{2, \Omega}+\|u(t)\|_{H^{4}\left(\Omega, \Gamma_{1}\right)} \\
& \quad \leq c\left[\left\|u^{1}\right\|_{2, \Omega}+\left\|u^{0}\right\|_{H^{4}\left(\Omega, \Gamma_{1}\right)}+\|f\|_{L^{1}\left(0, T^{\prime} ; H_{0}^{2}(\Omega)\right)}\right] .
\end{aligned}
$$


Theorem 1.4 can be proved by the variational methods (see [3], Chapter XVIII) and Lemma 1.2.

2. Boundary regularity. In this section we discuss the boundary regularity of the solution of (1.1). To this end, we first establish the following important identity that plays the key role in obtaining the boundary estimations for the solutions. These estimations are the essential part in the applications of HUM.

LEMma 2.1. Suppose that the boundary $\Gamma$ of $\Omega$ is of class $C^{3}$ and the boundary $\Gamma_{1}$ of $\Omega_{1}$ is of class $C^{4}$. Let $\rho=\left(\rho_{k}\right)$ be a vector field in $\left[C^{2}(\bar{\Omega})\right]^{n}$. Suppose that $u$ is the strong solution of (1.1). Then the following identity holds:

$$
\begin{aligned}
& \frac{1}{2} \int_{\Sigma} a_{2} \rho_{k} \nu_{k}\left|\Delta u_{2}\right|^{2} d \Sigma \\
&=\left.\left(u^{\prime}(t), \rho_{k} \frac{\partial u(t)}{\partial x_{k}}\right)\right|_{0} ^{T}+\frac{1}{2} \int_{Q} \frac{\partial \rho_{k}}{\partial x_{k}}\left[\left|u^{\prime}\right|^{2}-a(x)|\Delta u|^{2}\right] d x d t \\
& \quad+\int_{Q} a(x) \Delta \rho_{k} \Delta u \frac{\partial u}{\partial x_{k}} d x d t+2 \int_{Q} a(x) \frac{\partial \rho_{k}}{\partial x_{j}} \Delta u \frac{\partial^{2} u}{\partial x_{k} \partial x_{j}} d x d t \\
&-\int_{Q} f \rho_{k} \frac{\partial u}{\partial x_{k}} d x d t+\int_{Q} q \rho_{k} u \frac{\partial u}{\partial x_{k}} d x d t \\
&+\frac{1}{2} \int_{\Sigma_{1}} \rho_{k} \nu_{k}\left(a_{1}\left|\Delta u_{1}\right|^{2}-a_{2}\left|\Delta u_{2}\right|^{2}\right) d \Sigma+\int_{\Sigma_{1}} a_{1} \Delta u_{1} \rho_{k} \frac{\partial^{2}}{\partial \nu \partial x_{k}}\left(u_{2}-u_{1}\right) d \Sigma
\end{aligned}
$$

Proof. Multiplying (1.1) by $\rho_{k} \frac{\partial u}{\partial x_{k}}$ and integrating on $Q$, we have

$$
\int_{Q} \rho_{k} \frac{\partial u}{\partial x_{k}} u^{\prime \prime} d x d t+\int_{Q} \rho_{k} \frac{\partial u}{\partial x_{k}} a(x) \Delta^{2} u d x d t+\int_{Q} q \rho_{k} u \frac{\partial u}{\partial x_{k}} d x d t=\int_{Q} \rho_{k} \frac{\partial u}{\partial x_{k}} f d x d t
$$

Integrating by parts and noting that $a_{1} \frac{\partial \Delta u_{1}}{\partial \nu}=a_{2} \frac{\partial \Delta u_{2}}{\partial \nu}, \frac{\partial u_{1}}{\partial x_{k}}=\frac{\partial u_{2}}{\partial x_{k}}, a_{1} \Delta u_{1}=a_{2} \Delta u_{2}$ on $\Sigma_{1}$, and $\frac{\partial u_{2}}{\partial x_{k}}=0$ on $\Sigma$, we obtain

$$
\begin{aligned}
\int_{Q} \rho_{k} \frac{\partial u}{\partial x_{k}} u^{\prime \prime} d x d t= & \left.\left(u^{\prime}(t), \rho_{k} \frac{\partial u(t)}{\partial x_{k}}\right)\right|_{0} ^{T}-\frac{1}{2} \int_{\Sigma_{1}} \rho_{k} \nu_{k}\left|u_{1}^{\prime}\right|^{2} d \Sigma \\
& +\frac{1}{2} \int_{Q_{1}} \frac{\partial \rho_{k}}{\partial x_{k}}\left|u_{1}^{\prime}\right|^{2} d x d t+\frac{1}{2} \int_{\Sigma_{1}} \rho_{k} \nu_{k}\left|u_{2}^{\prime}\right|^{2} d \Sigma \\
& +\frac{1}{2} \int_{Q_{2}} \frac{\partial \rho_{k}}{\partial x_{k}}\left|u_{2}^{\prime}\right|^{2} d x d t \\
= & \left.\left(u^{\prime}(t), \rho_{k} \frac{\partial u(t)}{\partial x_{k}}\right)\right|_{0} ^{T}+\frac{1}{2} \int_{Q} \frac{\partial \rho_{k}}{\partial x_{k}}\left|u^{\prime}\right|^{2} d x d t
\end{aligned}
$$


and

$$
\begin{aligned}
\int_{Q} a(x) \rho_{k} & \frac{\partial u}{\partial x_{k}} \Delta^{2} u d x d t \\
= & \int_{\Sigma_{1}} a_{1} \frac{\partial \Delta u_{1}}{\partial \nu} \rho_{k} \frac{\partial u_{1}}{\partial x_{k}} d \Sigma-\int_{\Sigma_{1}} a_{1} \Delta u_{1} \frac{\partial}{\partial \nu}\left(\rho_{k} \frac{\partial u_{1}}{\partial x_{k}}\right) d \Sigma \\
& +\int_{Q_{1}} a_{1} \Delta u_{1} \Delta\left(\rho_{k} \frac{\partial u_{1}}{\partial x_{k}}\right) d x d t-\int_{\Sigma_{1}} a_{2} \frac{\partial \Delta u_{2}}{\partial \nu} \rho_{k} \frac{\partial u_{2}}{\partial x_{k}} d \Sigma \\
& +\int_{\Sigma_{1}} a_{2} \Delta u_{2} \frac{\partial}{\partial \nu}\left(\rho_{k} \frac{\partial u_{2}}{\partial x_{k}}\right) d \Sigma+\int_{Q_{2}} a_{2} \Delta u_{2} \Delta\left(\rho_{k} \frac{\partial u_{2}}{\partial x_{k}}\right) d x d t \\
& +\int_{\Sigma} a_{2} \frac{\partial \Delta u_{2}}{\partial \nu} \rho_{k} \frac{\partial u_{2}}{\partial x_{k}} d \Sigma-\int_{\Sigma} a_{2} \Delta u_{2} \frac{\partial}{\partial \nu}\left(\rho_{k} \frac{\partial u_{2}}{\partial x_{k}}\right) d \Sigma \\
= & \int_{\Sigma_{1}} a_{1} \Delta u_{1} \rho_{k} \frac{\partial^{2}}{\partial \nu \partial x_{k}}\left(u_{2}-u_{1}\right) d \Sigma-\int_{\Sigma} a_{2} \Delta u_{2} \rho_{k} \frac{\partial^{2} u_{2}}{\partial \nu \partial x_{k}} d \Sigma \\
& +\int_{Q_{1}} a_{1} \Delta u_{1} \Delta\left(\rho_{k} \frac{\partial u_{1}}{\partial x_{k}}\right) d x d t+\int_{Q_{2}} a_{2} \Delta u_{2} \Delta\left(\rho_{k} \frac{\partial u_{2}}{\partial x_{k}}\right) d x d t
\end{aligned}
$$

Moreover,

$$
\begin{aligned}
\int_{Q_{1}} a_{1} \Delta u_{1} \Delta\left(\rho_{k} \frac{\partial u_{1}}{\partial x_{k}}\right) d x d t+\int_{Q_{2}} a_{2} \Delta u_{2} \Delta\left(\rho_{k} \frac{\partial u_{2}}{\partial x_{k}}\right) d x d t \\
=\int_{Q} a(x)\left[\Delta \rho_{k} \Delta u \frac{\partial u}{\partial x_{k}}+2 \frac{\partial \rho_{k}}{\partial x_{j}} \Delta u \frac{\partial^{2} u}{\partial x_{k} \partial x_{j}}-\frac{1}{2} \frac{\partial \rho_{k}}{\partial x_{k}}|\Delta u|^{2}\right] d x d t \\
\quad+\frac{1}{2} \int_{\Sigma} a_{2} \rho_{k} \nu_{k}\left|\Delta u_{2}\right|^{2} d \Sigma+\frac{1}{2} \int_{\Sigma_{1}} \rho_{k} \nu_{k}\left(a_{1}\left|\Delta u_{1}\right|^{2}-a_{2}\left|\Delta u_{2}\right|^{2}\right) d \Sigma
\end{aligned}
$$

Noting that $\frac{\partial^{2} u_{2}}{\partial \nu \partial x_{k}}=\frac{\partial^{2} u_{2}}{\partial \nu^{2}} \nu_{k}$ and $\frac{\partial^{2} u_{2}}{\partial x_{k}^{2}}=\frac{\partial^{2} u_{2}}{\partial \nu^{2}} \nu_{k}^{2}$ on $\Sigma$, it therefore follows from (2.4) that

$$
\begin{aligned}
\int_{Q} a(x) & \rho_{k} \frac{\partial u}{\partial x_{k}} \Delta^{2} u d x d t \\
= & \int_{Q} a(x)\left[\Delta \rho_{k} \Delta u \frac{\partial u}{\partial x_{k}}+2 \frac{\partial \rho_{k}}{\partial x_{j}} \Delta u \frac{\partial^{2} u}{\partial x_{k} \partial x_{j}}-\frac{1}{2} \frac{\partial \rho_{k}}{\partial x_{k}}|\Delta u|^{2}\right] d x d t \\
& -\frac{1}{2} \int_{\Sigma} a_{2} \rho_{k} \nu_{k}\left|\Delta u_{2}\right|^{2} d \Sigma+\int_{\Sigma_{1}} a_{1} \Delta u_{1} \rho_{k} \frac{\partial^{2}}{\partial \nu \partial x_{k}}\left(u_{2}-u_{1}\right) d \Sigma \\
& +\frac{1}{2} \int_{\Sigma_{1}} \rho_{k} \nu_{k}\left(a_{1}\left|\Delta u_{1}\right|^{2}-a_{2}\left|\Delta u_{2}\right|^{2}\right) d \Sigma
\end{aligned}
$$


From (2.2), (2.3), and (2.6) we deduce

$$
\begin{aligned}
\int_{Q} f \rho_{k} \frac{\partial u}{\partial x_{k}} d x d t= & \left.\left(u^{\prime}(t), \rho_{k} \frac{\partial u(t)}{\partial x_{k}}\right)\right|_{0} ^{T}+\frac{1}{2} \int_{Q} \frac{\partial \rho_{k}}{\partial x_{k}}\left|u^{\prime}\right|^{2} d x d t \\
& +\int_{Q} a(x)\left[\Delta \rho_{k} \Delta u \frac{\partial u}{\partial x_{k}}+2 \frac{\partial \rho_{k}}{\partial x_{j}} \Delta u \frac{\partial^{2} u}{\partial x_{k} \partial x_{j}}-\frac{1}{2} \frac{\partial \rho_{k}}{\partial x_{k}}|\Delta u|^{2}\right] d x d t \\
& -\frac{1}{2} \int_{\Sigma} a_{2} \rho_{k} \cdot \nu_{k}\left|\Delta u_{2}\right|^{2} d \Sigma+\int_{\Sigma_{1}} a_{1} \Delta u_{1} \rho_{k} \frac{\partial^{2}}{\partial \nu \partial x_{k}}\left(u_{2}-u_{1}\right) d \Sigma \\
& +\frac{1}{2} \int_{\Sigma_{1}} \rho_{k^{\prime}} \nu_{k}\left(a_{1}\left|\Delta u_{1}\right|^{2}-a_{2}\left|\Delta u_{2}\right|^{2}\right) d \Sigma+\int_{Q} q \rho_{k} u \frac{\partial u}{\partial x_{k}} d x d t
\end{aligned}
$$

This implies (2.1).

After establishing the identity (2.1), we can obtain the boundary regularity and boundary estimations for the solution of (1.1).

Lemma 2.2. Suppose that the boundary $\Gamma$ of $\Omega$ is of class $C^{3}$. Then there exists a constant $c>0$ such that for all weak solutions of (1.1)

$$
\int_{\Sigma}\left|\Delta u_{2}\right|^{2} d \Sigma \leq c\left[\left\|u^{0}\right\|_{2 . \Omega}^{2}+\left\|u^{1}\right\|_{0 . \Omega}^{2}+\|f\|_{L^{1}\left(0 . T^{\prime}: L^{2}(\Omega)\right)}^{2}\right] .
$$

Proof. We choose $\rho \in\left[C^{2}(\bar{\Omega})\right]^{n}$ such that $\rho=\nu$ on $\Gamma$ and $\rho=0$ in $\Omega_{0}$, where the open set $\Omega_{0}$ satisfies $\bar{\Omega}_{1} \subset \Omega_{0} \subset \bar{\Omega}_{0} \subset \Omega$. Then the inequality (2.7) follows from Theorem 1.4 and the identity (2.1).

Lemma 2.3. Suppose that the boundaries $\Gamma$ and $\Gamma_{1}$ of $\Omega$ and $\Omega_{1}$ are of class $C^{4}$. Assume $q \in W^{1 . x}\left(0, T ; L^{\infty}(\Omega)\right) \cap L^{\infty}\left(0, T ; W^{2 \cdot x}(\Omega)\right)$. Then there exists a constant $c>0$ such that for all strong solutions of (1.1)

$$
\int_{\Sigma}\left|\Delta u_{2}^{\prime}\right|^{2} d \Sigma \leq c\left[\left\|u^{0}\right\|_{H^{4}\left(\Omega . \Gamma_{1}\right)}^{2}+\left\|u^{1}\right\|_{2 . \Omega}^{2}+\|f\|_{L^{1}\left(0 . T^{\prime}: H_{0}^{2}(\Omega)\right.}^{2}\right] .
$$

Proof. By density and passage to the limit, it suffices to prove $(2.8)$ for $f \in \mathcal{D}((0, T)$; $\left.H_{0}^{2}(\Omega)\right)$, where $\mathcal{D}\left((0, T) ; H_{0}^{2}(\Omega)\right)$ denotes the space of all infinitely differentiable functions in $t$ with compact supports in $(0, T)$ and values in $H_{0}^{2}(\Omega)$.

Let $u$ be the solution of (1.1) and set $v=u^{\prime}$. Then $v$ satisfies

$$
\begin{cases}v^{\prime \prime}+a(x) \Delta^{2} v+q v+q^{\prime}\left[u^{0}+\int_{0}^{t} v(s) d s\right]=f^{\prime} & \text { in } Q, \\ v(0)=u^{1}, \quad v^{\prime}(0)=-a(x) \Delta^{2} u^{0}-q u^{0} & \text { in } \Omega, \\ v_{2}=\frac{\partial v_{2}}{\partial \nu}=0 & \text { on } \Sigma, \\ v_{2}=v_{2}, \quad \frac{\partial v_{1}}{\partial \nu}=\frac{\partial v_{2}}{\partial \nu} & \text { on } \Sigma_{1}, \\ a_{1} \Delta v_{1}=a_{2} \Delta v_{2}, \quad a_{1} \frac{\partial \Delta v_{1}}{\partial \nu}=a_{2} \frac{\partial \Delta v_{2}}{\partial \nu} & \text { on } \Sigma_{1} .\end{cases}
$$


Choosing a vector field $\rho$ as in the proof of Lemma 2.2 and using the identity (2.1) (at this time, we have an additional term $\int_{Q} q^{\prime}\left[u^{0}+\int_{0}^{t} v(s) d s\right] \rho_{k} \frac{\partial v}{\partial x_{k}} d x d t$ ), we obtain

$$
\begin{aligned}
\frac{1}{2} \int_{\Sigma} a_{2}\left|\Delta u_{2}^{\prime}\right|^{2} d \Sigma= & \frac{1}{2} \int_{\Sigma} a_{2}\left|\Delta v_{2}\right|^{2} d \Sigma \\
= & \left.\left(v^{\prime}(t), \rho_{k} \frac{\partial v(t)}{\partial x_{k}}\right)\right|_{0} ^{T}+\frac{1}{2} \int_{Q} \frac{\partial \rho_{k}}{\partial x_{k}}\left[\left|v^{\prime}\right|^{2}-a(x)|\Delta v|^{2}\right] d x d t \\
& +\int_{Q} a(x) \Delta \rho_{k} \Delta v \frac{\partial v}{\partial x_{k}} d x d t \\
& +2 \int_{Q} a(x) \frac{\partial \rho_{k}}{\partial x_{j}} \Delta v \frac{\partial^{2} v}{\partial x_{k} \partial x_{j}} d x d t \\
& -\int_{Q} f^{\prime} \rho_{k} \frac{\partial v}{\partial x_{k}} d x d t+\int_{Q} q \rho_{k} v \frac{\partial v}{\partial x_{k}} d x d t \\
& +\int_{Q} q^{\prime}\left[u^{0}+\int_{0}^{t} v(s) d s\right] \rho_{k} \frac{\partial v}{\partial x_{k}} d x d t
\end{aligned}
$$

Since

$$
v^{\prime}=u^{\prime \prime}=f-a(x) \Delta^{2} u-q u
$$

we deduce

$$
\begin{aligned}
\int_{Q} \frac{d f}{d t} \rho_{k} \frac{\partial v}{\partial x_{k}} d x d t= & -\int_{Q} f \rho_{k} \frac{\partial v^{\prime}}{\partial x_{k}} d x d t \\
= & \int_{Q} v^{\prime} \frac{\partial}{\partial x_{k}}\left(f \rho_{k}\right) d x d t \\
= & \int_{Q} f \frac{\partial}{\partial x_{k}}\left(f \rho_{k}\right) d x d t-\int_{Q}\left(a(x) \Delta^{2} u+q u\right) \frac{\partial}{\partial x_{k}}\left(f \rho_{k}\right) d x d t \\
= & \int_{Q} f^{2} \frac{\partial \rho_{k}}{\partial x_{k}} d x d t+\frac{1}{2} \int_{Q} \rho_{k} \frac{\partial}{\partial x_{k}}\left(f^{2}\right) d x d t \\
& -\int_{Q}\left(a(x) \Delta^{2} u+q u\right) \frac{\partial}{\partial x_{k}}\left(f \rho_{k}\right) d x d t \\
= & \frac{1}{2} \int_{Q} f^{2} \frac{\partial \rho_{k}}{\partial x_{k}} d x d t-\int_{Q}\left(a(x) \Delta^{2} u+q u\right) \frac{\partial}{\partial x_{k}}\left(f \rho_{k}\right) d x d t .
\end{aligned}
$$

Substituting (2.11) and (2.12) into (2.10) yields 


$$
\begin{aligned}
& \frac{1}{2} \int_{\Sigma} a_{2}\left|\Delta u_{2}^{\prime}\right|^{2} d \Sigma \\
&=\left.\left(v^{\prime}(t), \rho_{k} \frac{\partial v(t)}{\partial x_{k}}\right)\right|_{0} ^{T}+\frac{1}{2} \int_{Q} \frac{\partial \rho_{k}}{\partial x_{k}}\left[\left|f-a(x) \Delta^{2} u-q u\right|^{2}-a(x)|\Delta v|^{2}\right] d x d t \\
&+\int_{Q} a(x) \Delta \rho_{k} \Delta v \frac{\partial v}{\partial x_{k}} d x d t+2 \int_{Q} a(x) \frac{\partial \rho_{k}}{\partial x_{j}} \Delta v \frac{\partial^{2} v}{\partial x_{k} \partial x_{j}} d x d t \\
&-\frac{1}{2} \int_{Q} f^{2} \frac{\partial \rho_{k}}{\partial x_{k}} d x d t+\int_{Q}\left(a(x) \Delta^{2} u+q u\right) \frac{\partial}{\partial x_{k}}\left(f \rho_{k}\right) d x d t \\
&+\int_{Q} q \rho_{k} v \frac{\partial v}{\partial x_{k}} d x d t+\int_{Q} q^{\prime}\left[u^{0}+\int_{0}^{t} v(s) d s\right] \rho_{k} \frac{\partial v}{\partial x_{k}} d x d t \\
&=\left.\left(v^{\prime}(t), \rho_{k} \frac{\partial v(t)}{\partial x_{k}}\right)\right|_{0} ^{T}+\int_{Q} a(x) \Delta \rho_{k} \Delta v \frac{\partial v}{\partial x_{k}} d x d t+2 \int_{Q} a(x) \frac{\partial \rho_{k}}{\partial x_{j}} \Delta v \frac{\partial^{2} v}{\partial x_{k} \partial x_{j}} d x d t \\
&+\frac{1}{2} \int_{Q} \frac{\partial \rho_{k}}{\partial x_{k}}\left[a^{2}(x)\left|\Delta^{2} u\right|^{2}+2 a q u \Delta^{2} u+q^{2} u^{2}-a(x)|\Delta v|^{2}\right] d x d t \\
&+\int_{Q}\left(a(x) \Delta^{2} u+q u\right) \rho_{k} \frac{\partial f}{\partial x_{k}} d x d t+\int_{Q} q \rho_{k} v \frac{\partial v}{\partial x_{k}} d x d t \\
&+\int_{Q} q^{\prime}\left[u^{0}+\int_{0}^{t} v(s) d s\right] \rho_{k} \frac{\partial v}{\partial x_{k}} d x d t .
\end{aligned}
$$

Since $f(0)=f(T)=0$, it follows from (2.13) and Theorem 1.4 that

$$
\int_{\Sigma}\left|\Delta u_{2}^{\prime}\right|^{2} d \Sigma \leq c\left[\left\|u^{0}\right\|_{H^{4}(\Omega, S)}^{2}+\left\|u^{1}\right\|_{2, \Omega}^{2}+\|f\|_{L^{1}\left(0, T ; H_{0}^{2}(\Omega)\right.}^{2}\right] .
$$

3. Nonhomogeneous boundary value problems. We are now in a position to consider the nonhomogeneous boundary value problem that introduces a control function $\phi \in L^{2}(\Sigma)$ :

$$
\begin{cases}u^{\prime \prime}+a(x) \Delta^{2} u+q u=0 & \text { in } Q, \\ u(0)=u^{0}, \quad u^{\prime}(0)=u^{1} & \text { in } \Omega, \\ u_{2}=0, \quad \frac{\partial u_{2}}{\partial \nu}=\phi & \text { on } \Sigma, \\ u_{1}=u_{2}, \quad \frac{\partial u_{1}}{\partial \nu}=\frac{\partial u_{2}}{\partial \nu} & \text { on } \Sigma_{1}, \\ a_{1} \Delta u_{1}=a_{2} \Delta u_{2}, \quad a_{1} \frac{\partial \Delta u_{1}}{\partial \nu}=a_{2} \frac{\partial \Delta u_{2}}{\partial \nu} & \text { on } \Sigma_{1} .\end{cases}
$$

The solution of (3.1) can be defined by the transposition method (see [12]) as follows.

Definition 3.1. $u$ is said to be an ultraweak solution of (3.1) for $\left(u^{0}, u^{1}\right) \in L^{2}(\Omega) \times$ $H^{-2}(\Omega)$ if we have

$$
\int_{Q} f u d x d t=\left\langle\theta(0), u^{1}\right\rangle-\int_{\Omega} \theta^{\prime}(0) u^{0} d x-\int_{\Sigma} a_{2} \phi \Delta \theta_{2} d \Sigma, \quad \forall f \in \mathcal{D}(Q),
$$


where $\theta=\theta(x, t)$ is the solution of

$$
\begin{cases}\theta^{\prime \prime}+a(x) \Delta^{2} \theta+q \theta=f & \text { in } Q, \\ \theta(T)=\theta^{\prime}(T)=0 & \text { in } \Omega, \\ \theta_{2}=\frac{\partial \theta_{2}}{\partial \nu}=0 & \text { on } \Sigma, \\ \theta_{1}=\theta_{2}, \quad \frac{\partial \theta_{1}}{\partial \nu}=\frac{\partial \theta_{2}}{\partial \nu} & \text { on } \Sigma_{1}, \\ a_{1} \Delta \theta_{1}=a_{2} \Delta \theta_{2}, \quad a_{1} \frac{\partial \Delta \theta_{1}}{\partial \nu}=a_{2} \frac{\partial \Delta \theta_{2}}{\partial \nu} & \text { on } \Sigma_{1} .\end{cases}
$$

and $\langle\cdot, \cdot\rangle$ denotes the dual product between the spaces $H_{0}^{2}(\Omega)$ and $H^{-2}(\Omega)$, and $\mathcal{D}(Q)$ denotes the space of all infinitely differentiable functions defined on $Q$ with compact supports in $Q$.

TheOREM 3.2. Suppose that the boundaries $\Gamma$ and $\Gamma_{1}$ are of class $C^{4}$. Assume that $q \in W^{1, \infty}\left(0, T ; W^{2, \infty}(\Omega)\right)$. Then for all $\left(u^{0}, u^{1}, \phi\right) \in L^{2}(\Omega) \times H^{-2}(\Omega) \times L^{2}(\Sigma)$, there exists a unique ultraweak solution of (3.1) with

$$
u \in C\left([0, T] ; L^{2}(\Omega)\right) \times C^{1}\left([0, T] ; H^{-2}(\Omega)\right) .
$$

Moreover, there exists $c>0$ such that for all $t \in[0, T]$

$$
\|u(t)\|_{0, \Omega}+\left\|u^{\prime}(t)\right\|_{-2, \Omega} \leq c\left[\left\|u^{0}\right\|_{0, \Omega}+\left\|u^{1}\right\|_{-2, \Omega}+\|\phi\|_{L^{2}(\Sigma)}\right] .
$$

Corollary 3.3. Let $\Gamma$ and $\Gamma_{1}$ and $q$ be as in Theorem 3.2. Then for all $\left(u^{0}, u^{1}, \phi, f\right) \in$ $L^{2}(\Omega) \times H^{-2}(\Omega) \times L^{2}(\Sigma) \times L^{1}\left(0, T ; L^{2}(\Omega)\right)$, there exists a unique ultraweak solution of

$$
\begin{cases}u^{\prime \prime}+a(x) \Delta^{2} u+q u=f & \text { in } Q, \\ u(0)=u^{0}, \quad u^{\prime}(0)=u^{1} & \text { in } \Omega, \\ u_{2}=0, \quad \frac{\partial u_{2}}{\partial \nu}=\phi & \text { on } \Sigma, \\ u_{1}=u_{2}, \quad \frac{\partial u_{1}}{\partial \nu}=\frac{\partial u_{2}}{\partial \nu} & \text { on } \Sigma_{1}, \\ a_{1} \Delta u_{1}=a_{2} \Delta u_{2}, \quad a_{1} \frac{\partial \Delta u_{1}}{\partial \nu}=a_{2} \frac{\partial \Delta u_{2}}{\partial \nu} & \text { on } \Sigma_{1},\end{cases}
$$

with

$$
u \in C\left([0, T] ; L^{2}(\Omega)\right) \times C^{1}\left([0, T] ; H^{-2}(\Omega)\right) .
$$

Moreover, there exists $c>0$ such that for all $t \in[0, T]$

$$
\begin{aligned}
& \|u(t)\|_{0, \Omega}+\left\|u^{\prime}(t)\right\|_{-2, \Omega} \\
& \quad \leq c\left[\left\|u^{0}\right\|_{0, \Omega}+\left\|u^{1}\right\|_{-2, \Omega}+\|\phi\|_{L^{2}(\Sigma)}+\|f\|_{L^{1}\left(0, T ; L^{2}(\Omega)\right)}\right] .
\end{aligned}
$$

To prove Theorem 3.2, the following lemmas are necessary.

Lemma 3.4. Suppose that the boundaries $\Gamma$ and $\Gamma_{1}$ are of class $C^{4}$. Assume that $q \in$ $W^{1, \infty}\left(0, T ; W^{2, \infty}(\Omega)\right)$. If $f=\frac{d g}{d t}$ with $g \in L^{1}\left(0, T ; H_{0}^{2}(\Omega)\right)$, then there exists a constant $c>0$ such that for all solutions of (1.1) with $u^{0}=u^{1}=0$

$$
\|u(T)\|_{2, \Omega}+\left\|u^{\prime}(T)\right\|_{0, \Omega}+\left\|\Delta u_{2}\right\|_{L^{2}(\Sigma)} \leq c\|g\|_{L^{1}\left(0, T ; H_{0}^{2}(\Omega)\right)} .
$$


Proof. By density and passage to the limit, it suffices to prove $(3.9)$ for $g \in \mathcal{D}((0, T)$; $\left.H_{0}^{2}(\Omega)\right)$.

Let $w$ be the solution of

$$
\begin{cases}w^{\prime \prime}+a(x) \Delta^{2} w+q w-\int_{0}^{t} q^{\prime}(x, s) w(x, s) d x=g & \text { in } Q \\ w(0)=w^{\prime}(0)=0 & \text { in } \Omega \\ w_{2}=\frac{\partial w_{2}}{\partial \nu}=0 & \text { on } \Sigma \\ w_{1}=w_{2}, \quad \frac{\partial w_{1}}{\partial \nu}=\frac{\partial w_{2}}{\partial \nu} & \text { on } \Sigma_{1} \\ a_{1} \Delta w_{1}=a_{2} \Delta w_{2}, \quad a_{1} \frac{\partial \Delta w_{1}}{\partial \nu}=a_{2} \frac{\partial \Delta w_{2}}{\partial \nu} & \text { on } \Sigma_{1} .\end{cases}
$$

Then $u=w^{\prime}$ is the solution of (1.1) with $u^{0}=u^{1}=0$ since $u^{\prime}(0)=w^{\prime \prime}(0)=g(0)-$ $a(x) \Delta^{2} w(0)-q w(0)=0$ (note that $g(0)=0$ ). (3.10) is solvable because $w=\int_{0}^{t} u(s) d s$. Similar to (1.12) and (1.14), we deduce that there is a constant $c>0$ such that

$$
\begin{gathered}
\|w(t)\|_{2 . \Omega 2}+\left\|w^{\prime}(t)\right\|_{0 . \Omega} \leq c\|g\|_{L^{1}\left(0 . T ; L^{2}(\Omega)\right)}, \quad \forall t \in[0, T], \\
\left\|\Delta w^{\prime}(t)\right\|_{0, \Omega 2}+\left\|\Delta^{2} w(t)\right\|_{0 . \Omega} \leq c\|g\|_{L^{1}\left(0, T: H_{0}^{2}(\Omega)\right)}, \quad \forall t \in[0, T] .
\end{gathered}
$$

Since

$$
u=w^{\prime} \quad \text { and } \quad u^{\prime}(T)=w^{\prime \prime}(T)=-a(x) \Delta^{2} w(T)-q w(T)+\int_{0}^{T^{\prime}} q^{\prime}(x, s) w(x, s) d x
$$

(because $g(T)=0$ ), it follows from (3.11) and (3.12) that

$$
\|u(t)\|_{2, \Omega}+\left\|\Delta^{2} w(t)\right\|_{0, \Omega} \leq c\|g\|_{L^{1}\left(0, T ; H_{0}^{2}(\Omega)\right)}, \quad \forall t \in[0, T]
$$

and

$$
\begin{aligned}
& \|u(T)\|_{2, \Omega \Omega}+\left\|u^{\prime}(T)\right\|_{0 . \Omega} \\
& \quad \leq c\left(\|g\|_{L^{1}\left(0, T^{\prime}: H_{0}^{2}(\Omega)\right)}+\left\|w(T)+\int_{0}^{T} q^{\prime}(x, s) w(x, s) d s\right\|_{0, \Omega}\right) \\
& \quad \leq c\|g\|_{L^{1}\left(0, T^{\prime} ; H_{0}^{2}(\Omega)\right)} .
\end{aligned}
$$

On the other hand, choosing $\rho \in\left[C^{2}(\bar{\Omega})\right]^{n}$ such that $\rho=\nu$ on $\Gamma$ and $\rho=0$ in $\Omega_{0}$, where the open set $\Omega_{0}$ satisfies $\bar{\Omega}_{1} \subset \Omega_{0} \subset \bar{\Omega}_{0} \subset \Omega$, and applying (2.1), we obtain

$$
\begin{aligned}
\frac{1}{2} \int_{\Sigma} a_{2}\left|\Delta u_{2}\right|^{2} d \Sigma \\
=\left(u^{\prime}(T), \rho_{k} \frac{\partial u(T)}{\partial x_{k}}\right)+\frac{1}{2} \int_{Q} \frac{\partial \rho_{k}}{\partial x_{k}}\left[\left|u^{\prime}\right|^{2}-a(x)|\Delta u|^{2}\right] d x d t \\
\quad+2 \int_{Q} a(x) \frac{\partial \rho_{k}}{\partial x_{j}} \Delta u \frac{\partial^{2} u}{\partial x_{k} \partial x_{j}} d x d t+\int_{Q} a(x) \Delta \rho_{k} \Delta u \frac{\partial u}{\partial x_{k}} d x d t \\
\quad-\int_{Q} \frac{d g}{d t} \rho_{k} \frac{\partial u}{\partial x_{k}} d x d t+\int_{Q} q \rho_{k} u \frac{\partial u}{\partial x_{k}} d x d t
\end{aligned}
$$


Since

$$
u^{\prime}=w^{\prime \prime}=g-a(x) \Delta^{2} w-q w+\int_{0}^{t} q^{\prime}(x, s) w(x, s) d s
$$

we deduce in the same manner as in (2.12)

$$
\begin{aligned}
\int_{Q} \frac{d g}{d t} \rho_{k} & \frac{\partial u}{\partial x_{k}} d x d t \\
= & \frac{1}{2} \int_{Q} g^{2} \frac{\partial \rho_{k}}{\partial x_{k}} d x d t-\int_{Q}\left(a(x) \Delta^{2} w+q w\right) \frac{\partial}{\partial x_{k}}\left(g \rho_{k}\right) d x d t \\
& +\int_{Q} \frac{\partial}{\partial x_{k}}\left(g \rho_{k}\right) \int_{0}^{t} q^{\prime}(x, s) w(x, s) d s d x d t
\end{aligned}
$$

Substituting (3.16) and (3.17) into (3.15) yields

$$
\begin{aligned}
\frac{1}{2} \int_{\Sigma} a_{2}\left|\Delta u_{2}\right|^{2} d \Sigma \\
=\left(u^{\prime}(T), \rho_{k} \frac{\partial u(T)}{\partial x_{k}}\right)+\int_{Q} a(x) \Delta \rho_{k} \Delta u \frac{\partial u}{\partial x_{k}} d x d t+\int_{Q} q \rho_{k} u \frac{\partial u}{\partial x_{k}} d x d t \\
+\frac{1}{2} \int_{Q} \frac{\partial \rho_{k}}{\partial x_{k}}\left[a^{2}(x)\left|\Delta^{2} w\right|^{2}+2 q a w \Delta^{2} w+q^{2} w^{2}-a(x)|\Delta u|^{2}\right] d x d t \\
+\int_{Q} \frac{\partial \rho_{k}}{\partial x_{k}}\left(g-a(x) \Delta^{2} w-q w\right) \int_{0}^{t} q^{\prime}(x, s) w(x, s) d s d x d t \\
+\frac{1}{2} \int_{Q} \frac{\partial \rho_{k}}{\partial x_{k}}\left(\int_{0}^{t} q^{\prime}(x, s) w(x, s) d s\right)^{2} d x d t \\
+2 \int_{Q} a(x) \frac{\partial \rho_{k}}{\partial x_{j}} \Delta u \frac{\partial^{2} u}{\partial x_{k} \partial x_{j}} d x d t+\int_{Q}\left(a(x) \Delta^{2} w+q w\right) \rho_{k} \frac{\partial g}{\partial x_{k}} d x d t \\
\quad-\int_{Q} \frac{\partial}{\partial x_{k}}\left(g \rho_{k}\right) \int_{0}^{t} q^{\prime}(x, s) w(x, s) d s d x d t .
\end{aligned}
$$

It therefore follows from (3.13), (3.14), and (3.18) that

$$
\left\|\Delta u_{2}\right\|_{L^{2}(\Sigma)} \leq c\|g\|_{L^{1}\left(0, T ; H_{0}^{2}(\Omega)\right)} .
$$

Finally, (3.9) follows from (3.14) and (3.19).

Lemma 3.5. Let $\Gamma$ and $\Gamma_{1}$ be of class $C^{4}$. Then for

$$
\phi_{j} \in H^{4-j-\frac{1}{2}}(\Gamma), \quad j=0,1,2,3
$$

there exists $u \in H^{4}\left(\Omega_{1}, \Omega_{2}\right)$ such that

$$
\frac{\partial^{j} u}{\partial \nu^{j}}=\phi_{j}, \quad \text { on } \Gamma, \quad j=0,1,2,3
$$


where

$$
\begin{aligned}
& H^{4}\left(\Omega_{1}, \Omega_{2}\right)=\left\{u: u \in H^{2}(\Omega) ; u_{i} \in H^{4}\left(\Omega_{i}\right), i=1,2 ;\right. \\
& \left.a_{1} \Delta u_{1}=a_{2} \Delta u_{2} \text { and } a_{1} \frac{\partial \Delta u_{1}}{\partial \nu}=a_{2} \frac{\partial \Delta u_{2}}{\partial \nu} \text { on } \Gamma_{1}\right\} \text {. }
\end{aligned}
$$

Proof. By the trace theorem (see [12], Chapter 1), it follows that there exists $w \in$ $H^{4}(\Omega)$ such that

$$
\frac{\partial^{j} w}{\partial \nu^{j}}=\phi_{j}, \quad \text { on } \Gamma, \quad j=0,1,2,3 .
$$

Likewise, there exists $v \in H^{4}\left(\Omega_{1}\right)$ such that

$$
\begin{gathered}
v=w \quad \text { on } \Gamma_{1} \\
\frac{\partial v}{\partial \nu}=\frac{\partial w}{\partial \nu} \quad \text { on } \Gamma_{1} \\
\Delta v=\frac{a_{2}}{a_{1}} \Delta w \quad \text { on } \Gamma_{1}, \\
\frac{\partial \Delta v}{\partial \nu}=\frac{a_{2}}{a_{1}} \frac{\partial \Delta w}{\partial \nu} \quad \text { on } \Gamma_{1}
\end{gathered}
$$

Then, $u$ defined by

$$
u= \begin{cases}v, & x \in \Omega_{1} \\ w, & x \in \Omega_{2}\end{cases}
$$

belongs to $H^{4}\left(\Omega_{1}, \Omega_{2}\right)$ and satisfies

$$
\frac{\partial^{j} u}{\partial \nu^{j}}=\phi_{j}, \quad \text { on } \Gamma, \quad j=0,1,2,3 . \quad \odot
$$

We are now ready to prove Theorem 3.2 .

Proof of Theorem 3.2. It follows from Definition 3.1, Theorem 1.4, and Lemma 2.2 that

$$
\begin{aligned}
\left|\int_{Q} u f d x d t\right| \leq & c\left[\left\|u^{0}\right\|_{0, \Omega}+\left\|u^{1}\right\|_{-2, \Omega}+\|\phi\|_{L^{2}(\Sigma)}\right] \\
& \times\left[\|\theta(0)\|_{2, \Omega}+\left\|\theta^{\prime}(0)\right\|_{0, \Omega}+\left\|\Delta \theta_{2}\right\|_{L^{2}(\Sigma)}\right] \\
\leq & c\left[\left\|u^{0}\right\|_{0, \Omega}+\left\|u^{1}\right\|_{-2, \Omega}+\|\phi\|_{L^{2}(\Sigma)}\right]\|f\|_{L^{1}\left(0, T ; L^{2}(\Omega)\right)} .
\end{aligned}
$$

Therefore, there exists a $u \in L^{\infty}\left(0, T ; L^{2}(\Omega)\right)$ such that (3.2) holds. Moreover,

$$
\|u\|_{L^{\infty}\left(0, T ; L^{2}(\Omega)\right)} \leq c\left[\left\|u^{0}\right\|_{0, \Omega}+\left\|u^{1}\right\|_{-2, \Omega}+\|\phi\|_{L^{2}(\Sigma)}\right] .
$$

On the other hand, if $f=\frac{d g}{d t}$ with $g \in L^{1}\left(0, T ; H_{0}^{2}(\Omega)\right)$, then Lemma 3.4 gives

$$
\left|\int_{Q} u \frac{d g}{d t} d x d t\right| \leq c\left[\left\|u^{0}\right\|_{0, \Omega}+\left\|u^{1}\right\|_{-2, \Omega}+\|\phi\|_{L^{2}(\Sigma)}\right]\|g\|_{L^{1}\left(0, T ; H_{0}^{2}(\Omega)\right)}
$$


which implies that $u^{\prime} \in L^{\infty}\left(0, T ; H^{-2}(\Omega)\right)$, and

$$
\left\|u^{\prime}\right\|_{L^{\infty}\left(0, T ; H^{-2}(\Omega)\right)} \leq c\left[\left\|u^{0}\right\|_{0, \Omega}+\left\|u^{1}\right\|_{-2, \Omega}+\|\phi\|_{L^{2}(\Sigma)}\right] .
$$

Hence, if we can prove

$$
u \in C\left([0, T] ; L^{2}(\Omega)\right) \cap C^{1}\left([0, T] ; H^{-2}(\Omega)\right)
$$

for sufficiently regular $u^{0}, u^{1}$, and $\phi$, then by the density, the theorem follows from (3.31) and (3.33). In doing so, we may as well assume that $\left(u^{0}, u^{1}\right) \in H_{0}^{2}(\Omega) \times L^{2}(\Omega)$ and $\phi \in \mathcal{D}\left((0, T) ; H^{\frac{5}{2}}(\Gamma)\right)$. By Lemma 3.5 , there is $\psi \in \mathcal{D}\left((0, T) ; H^{4}\left(\Omega_{1}, \Omega_{2}\right) \cap H_{0}^{1}(\Omega)\right)$ such that

$$
\frac{\partial \psi}{\partial \nu}=\phi, \quad \text { on } \Sigma
$$

Set

$$
v=u-\psi
$$

Then

$$
\begin{cases}v^{\prime \prime}+a(x) \Delta^{2} v+q v=-\left(\psi^{\prime \prime}+a(x) \Delta^{2} \psi+q \psi\right)=F & \text { in } Q, \\ v(0)=u^{0}, \quad v^{\prime}(0)=u^{1} & \text { in } \Omega, \\ v_{2}=\frac{\partial v_{2}}{\partial \nu}=0 & \text { on } \Sigma, \\ v_{1}=v_{2}, \quad \frac{\partial v_{1}}{\partial \nu}=\frac{\partial v_{2}}{\partial \nu} & \text { on } \Sigma_{1} \\ a_{1} \Delta v_{1}=a_{2} \Delta v_{2}, \quad a_{1} \frac{\partial \Delta v_{1}}{\partial \nu}=a_{2} \frac{\partial \Delta v_{2}}{\partial \nu} & \text { on } \Sigma_{1} .\end{cases}
$$

It is clear that $F \in L^{1}\left(0, T ; L^{2}(\Omega)\right)$. Thus, it follows from Theorem 1.4 that

$$
v \in C\left([0, T] ; H_{0}^{2}(\Omega)\right) \cap C^{1}\left([0, T] ; L^{2}(\Omega)\right) .
$$

Consequently,

$$
\begin{aligned}
u= & v+\psi \in C\left([0, T] ; H^{2}(\Omega)\right) \cap C^{1}\left([0, T] ; L^{2}(\Omega)\right) \\
& \subset C\left([0, T] ; L^{2}(\Omega)\right) \cap C^{1}\left([0, T] ; H^{-2}(\Omega)\right) .
\end{aligned}
$$

We now consider the nonhomogeneous boundary value problem (3.1) in the case where $\phi \in\left(H^{1}\left(0, T ; L^{2}(\Gamma)\right)^{\prime}\right.$, i.e., $\phi=\frac{\partial \xi}{\partial t}$ with $\xi \in L^{2}(\Sigma)$. Therefore, problem (3.1) now becomes

$$
\begin{cases}u^{\prime \prime}+a(x) \Delta^{2} u+q u=0 & \text { in } Q, \\ u(0)=u^{0}, \quad u^{\prime}(0)=u^{1} & \text { in } \Omega, \\ u_{2}=0, \quad \frac{\partial u_{2}}{\partial \nu}=\frac{\partial \xi}{\partial t} & \text { on } \Sigma, \\ u_{1}=u_{2}, \quad \frac{\partial u_{1}}{\partial \nu}=\frac{\partial u_{2}}{\partial \nu} & \text { on } \Sigma_{1}, \\ a_{1} \Delta u_{1}=a_{2} \Delta u_{2}, \quad a_{1} \frac{\partial \Delta u_{1}}{\partial \nu}=a_{2} \frac{\partial \Delta u_{2}}{\partial \nu} & \text { on } \Sigma_{1} .\end{cases}
$$


This problem can be solved in a manner similar to the above. To this end, let $\left(\theta^{0}, \theta^{1}, f\right) \in$ $H^{4}\left(\Omega, \Gamma_{1}\right) \times H_{0}^{2}(\Omega) \times L^{1}\left(0, T ; H_{0}^{2}(\Omega)\right)$ and $\theta=\theta(x, t)$ be the solution of

$$
\begin{cases}\theta^{\prime \prime}+a(x) \Delta^{2} \theta+q \theta=f & \text { in } Q, \\ \theta(T)=\theta^{0}, \quad \theta^{\prime}(T)=\theta^{1} & \text { in } \Omega, \\ \theta_{2}=\frac{\partial \theta_{2}}{\partial \nu}=0 & \text { on } \Sigma, \\ \theta_{1}=\theta_{2}, \quad \frac{\partial \theta_{1}}{\partial \nu}=\frac{\partial \theta_{2}}{\partial \nu} & \text { on } \Sigma_{1}, \\ a_{1} \Delta \theta_{1}=a_{2} \Delta \theta_{2}, \quad a_{1} \frac{\partial \Delta \theta_{1}}{\partial \nu}=a_{2} \frac{\partial \Delta \theta_{2}}{\partial \nu} & \text { on } \Sigma_{1} .\end{cases}
$$

Multiplying the first equation of (3.41) by $u$ and formally integrating by parts over $Q$, we obtain

$$
\int_{Q} f u d x d t+\left\langle\theta^{0}, u^{\prime}(T)\right\rangle-\left\langle\theta^{1}, u(T)\right\rangle=\left\langle\theta(0), u^{1}\right\rangle-\left\langle\theta^{\prime}(0), u^{0}\right\rangle-\int_{\Sigma} a_{2} \frac{\partial \xi}{\partial t} \Delta \theta_{2} d \Sigma
$$

Here and in the sequel $\langle\cdot, \cdot\rangle$ denote various dual products between a space and its dual such as $H_{0}^{2}(\Omega)$ and $H^{-2}(\Omega)$. On the other hand, in the sense of derivatives in $\left(H^{1}\left(0, T ; L^{2}(\Gamma)\right)\right)^{\prime}$, we have

$$
\int_{\Sigma} a_{2} \frac{\partial \xi}{\partial t} \Delta \theta_{2} d \Sigma=-\int_{\Sigma} a_{2} \xi \Delta \theta_{2}^{\prime} d \Sigma
$$

It therefore follows that

$$
\int_{Q} f u d x d t+\left\langle\theta^{0}, u^{\prime}(T)\right\rangle-\left\langle\theta^{1}, u(T)\right\rangle=\left\langle\theta(0), u^{1}\right\rangle-\left\langle\theta^{\prime}(0), u^{0}\right\rangle+\int_{\Sigma} a_{2} \xi \Delta \theta_{2}^{\prime} d \Sigma
$$

Set

$$
L\left(\theta^{0}, \theta^{1}, f\right)=\left\langle\theta(0), u^{1}\right\rangle-\left\langle\theta^{\prime}(0), u^{0}\right\rangle+\int_{\Sigma} a_{2} \xi \Delta \theta_{2}^{\prime} d \Sigma .
$$

It follows from Theorem 1.4 and Lemma 2.3 that

$$
\begin{aligned}
\left|L\left(\theta^{0}, \theta^{1}, f\right)\right| \leq & \|\theta(0)\|_{H^{4}\left(\Omega, \Gamma_{1}\right)}\left\|u^{1}\right\|_{\left(H^{4}\left(\Omega, \Gamma_{1}\right)\right)^{\prime}}+\left\|\theta^{\prime}(0)\right\|_{2, \Omega}\left\|u^{0}\right\|_{-2, \Omega} \\
& +a_{2}\|\xi\|_{L^{2}(\Sigma)} \times\left\|\Delta \theta_{2}^{\prime}\right\|_{L^{2}(\Sigma)} \\
\leq & c\left[\left\|u^{0}\right\|_{-2, \Omega}+\left\|u^{1}\right\|_{\left(H^{4}\left(\Omega, \Gamma_{1}\right)\right)^{\prime}}+\|\xi\|_{L^{2}(\Sigma)}\right] \\
& \times\left[\left\|\theta^{0}\right\|_{H^{4}\left(\Omega, \Gamma_{1}\right)}+\left\|\theta^{1}\right\|_{2, \Omega}+\|f\|_{L^{1}\left(0, T ; H_{0}^{2}(\Omega)\right)}\right] .
\end{aligned}
$$

This shows that $L$ is a linear continuous functional on $H^{4}\left(\Omega, \Gamma_{1}\right) \times H_{0}^{2}(\Omega) \times L^{1}(0, T$; $\left.H_{0}^{2}(\Omega)\right)$. Hence there exists $u \in L^{\infty}\left(0, T ; H^{-2}(\Omega)\right)$ and $\left(\psi^{0}, \psi^{1}\right) \in H^{-2}(\Omega) \times\left(H^{4}\left(\Omega, \Gamma_{1}\right)\right)^{\prime}$ such that

$$
\langle u, f\rangle+\left\langle\psi^{1}, \theta^{0}\right\rangle+\left\langle-\psi^{0}, \theta^{1}\right\rangle=L\left(\theta^{0}, \theta^{1}, f\right)
$$

for all $\left(\theta^{0}, \theta^{1}, f\right) \in H^{4}\left(\Omega, \Gamma_{1}\right) \times H_{0}^{2}(\Omega) \times L^{1}\left(0, T ; H_{0}^{2}(\Omega)\right.$. Moreover,

$$
\begin{aligned}
& \|u\|_{L^{\infty}\left(0, T^{\prime} ; H^{-2}(\Omega)\right)}+\left\|\psi^{0}\right\|_{-2, \Omega}+\left\|\psi^{1}\right\|_{\left(H^{4}\left(\Omega, \Gamma_{1}\right)\right)^{\prime}} \\
& \quad \leq C\left[\left\|u^{0}\right\|_{-2, \Omega}+\left\|u^{1}\right\|_{\left(H^{4}\left(\Omega, \Gamma_{1}\right)\right)^{\prime}}+\|\xi\|_{L^{2}(\Sigma)}\right] .
\end{aligned}
$$

The above calculations lead to 
Definition 3.6. The function $u$ that satisfies (3.47) is said to be an ultraweak solution of the problem (3.40), and $\psi^{0}, \psi^{1}$ that satisfy (3.47) are defined to be values of $u, u^{\prime}$ at $T$, respectively, i.e.,

$$
u(T)=\psi^{0}, \quad u^{\prime}(T)=\psi^{1}
$$

Of course, we need to legitimatize Definition 3.6 a little bit. We do so in the case that $q$ is independent of $t$. The following procedure is not applicable for the case that $q$ depends on $t$. We can arrive at this point by carefully choosing particular "test functions" $\theta^{0}, \theta^{1}$, and $f$. In doing so, we introduce the eigenfunction $w$ of $a(x) \Delta^{2}+q$ :

$$
\begin{cases}a(x) \Delta^{2} w+q w=\lambda w & \text { in } \Omega \\ w_{2}=\frac{\partial w_{2}}{\partial \nu}=0 & \text { on } \Gamma \\ w_{1}=w_{2}, \quad \frac{\partial w_{1}}{\partial \nu}=\frac{\partial w_{2}}{\partial \nu} & \text { on } \Gamma_{1} \\ a_{1} \Delta w_{1}=a_{2} \Delta w_{2}, \quad a_{1} \frac{\partial \Delta w_{1}}{\partial \nu}=a_{2} \frac{\partial \Delta w_{2}}{\partial \nu} & \text { on } \Gamma_{1} .\end{cases}
$$

We take

$$
f=h(t) w
$$

Then

$$
\theta=p(t) w
$$

with

$$
p^{\prime \prime}+\lambda p=h .
$$

Substituting (3.51) and (3.52) into (3.47), we obtain

$$
\begin{aligned}
& \int_{0}^{T}\langle u, w\rangle\left(p^{\prime \prime}+\lambda p\right) d t+\left\langle\psi^{1}, w\right\rangle p(T)-\left\langle\psi^{0}, w\right\rangle p^{\prime}(T) \\
& \quad=\left\langle u^{1}, w\right\rangle p(0)-\left\langle u^{0}, w\right\rangle p^{\prime}(0)+\int_{\Sigma} a_{2} \xi p^{\prime} \Delta w_{2} d \Sigma
\end{aligned}
$$

Taking $p(0)=p^{\prime}(0)=p(T)=p^{\prime}(T)=0$, we get

$$
\langle u, w\rangle^{\prime \prime}+\lambda\langle u, w\rangle=-\int_{\Gamma} a_{2} \xi^{\prime} \Delta w_{2} d \Gamma,
$$

where derivatives $\langle u, w\rangle^{\prime \prime}$ and $\xi^{\prime}$ are understood in the sense of duals $\left(H^{2}(0, T)\right)^{\prime}$ and $\left(H^{1}(0, T) ; L^{2}(\Gamma)\right)^{\prime}$, respectively. It therefore follows from (3.54) that

$$
\left.\langle u, w\rangle p^{\prime}\right|_{0} ^{T}-\left.\langle u, w\rangle^{\prime} p\right|_{0} ^{T}+\left\langle\psi^{1}, w\right\rangle p(T)-\left\langle\psi^{0}, w\right\rangle p^{\prime}(T)=\left\langle u^{1}, w\right\rangle p(0)-\left\langle u^{0}, w\right\rangle p^{\prime}(0) .
$$

By taking $p(0) \neq 0$ and $p^{\prime}(0)=p(T)=p^{\prime}(T)=0$, we get

$$
\left\langle u^{1}, w\right\rangle=\langle u, w\rangle^{\prime}(0) \text {. }
$$

Likewise, we obtain

$$
\left\langle u^{0}, w\right\rangle=\langle u, w\rangle(0), \quad\left\langle\psi^{0}, w\right\rangle=\langle u, w\rangle(T), \quad\left\langle\psi^{1}, w\right\rangle=\langle u, w\rangle^{\prime}(T) .
$$


Thus

$$
u(0)=u^{0}, \quad u^{\prime}(0)=u^{1}, \quad u(T)=\psi^{0}, \quad u^{\prime}(T)=\psi^{1}
$$

hold in the sense of (3.57) and (3.58).

In addition, as in the proof of Theorem 3.2, by a density argument, we can show that

$$
u \in C\left([0, T] ; H^{-2}(\Omega)\right) .
$$

So far we have proved the following.

TheOREm 3.7. Suppose that the boundaries $\Gamma$ and $\Gamma_{1}$ are of class $C^{4}$. Assume that $q \in W^{1, \infty}\left(0, T ; W^{2, \infty}(\Omega)\right)$. Then for all $\left(u^{0}, u^{1}, \xi\right) \in H^{-2}(\Omega) \times\left(H^{4}\left(\Omega, \Gamma_{1}\right)\right)^{\prime} \times L^{2}(\Sigma)$, there exists a unique ultraweak solution of (3.40) in the sense of Definition 3.6 with

$$
\begin{gathered}
u \in C\left([0, T] ; H^{-2}(\Omega)\right), \\
u(T) \in H^{-2}(\Omega), \quad u^{\prime}(T) \in\left(H^{4}\left(\Omega, \Gamma_{1}\right)\right)^{\prime} .
\end{gathered}
$$

Moreover, there exists $c>0$ such that

$$
\begin{array}{r}
\|u\|_{L^{\infty}\left(0, T ; H^{-2}(\Omega)\right)}+\left\|\psi^{0}\right\|_{-2, \Omega}+\left\|\psi^{1}\right\|_{\left(H^{4}\left(\Omega, \Gamma_{1}\right)\right)^{\prime}} \\
\leq c\left[\left\|u^{0}\right\|_{-2, \Omega}+\left\|u^{1}\right\|_{\left(H^{4}\left(\Omega, \Gamma_{1}\right)\right)^{\prime}}+\|\xi\|_{L^{2}(\Sigma)}\right] .
\end{array}
$$

It is known from Lemmas 2.2 and 2.3 that $\left.\Delta u_{2}\right|_{L^{2}(\Sigma)} \in L^{2}(\Sigma)$ for the solutions $u$ of $(1.1)$ with $\left(u^{0}, u^{1}, f\right) \in H_{0}^{2}(\Omega) \times L^{2}(\Omega) \times L^{1}\left(0, T ; L^{2}(\Omega)\right)$ and $\left.\Delta u_{2}^{\prime}\right|_{L^{2}(\Sigma)} \in L^{2}(\Sigma)$ if $\left(u^{0}, u^{1}, f\right) \in H^{4}\left(\Omega, \Gamma_{1}\right) \times H_{0}^{2}(\Omega) \times L^{1}\left(0, T ; H_{0}^{2}(\Omega)\right)$. We are now interested in the boundary regularity for the ultraweak solutions of $(1.1)$ with $\left(u^{0}, u^{1}, f\right) \in L^{2}(\Omega) \times H^{-2}(\Omega) \times$ $L^{2}\left(0, T ; L^{2}(\Omega)\right)$. In this case the ultraweak solutions of (1.1) are guaranteed by Corollary 3.3 .

Lemma 3.8. Suppose that the boundaries $\Gamma$ and $\Gamma_{1}$ are of class $C^{4}$. Assume that $q \in$ $W^{1, \infty}\left(0, T ; W^{2, \infty}(\Omega)\right)$. Then there exists a constant $c>0$ such that for all ultraweak solutions of $(1.1)$ with $\left(u^{0}, u^{1}, f\right) \in L^{2}(\Omega) \times H^{-2}(\Omega) \times L^{2}\left(0, T ; L^{2}(\Omega)\right)$

$$
\left\|\Delta u_{2}\right\|_{H^{-1}\left(0, T ; L^{2}(\Gamma)\right)}^{2} \leq c\left[\left\|u^{1}\right\|_{-2, \Omega}^{2}+\left\|u^{0}\right\|_{0, \Omega}^{2}+\|f\|_{L^{2}\left(0, T ; L^{2}(\Omega)\right)}^{2}\right] .
$$

Proof. We introduce the solution $e$ of

$$
\begin{cases}a(x) \Delta^{2} e=-u^{1} & \text { in } \Omega, \\ e_{2}=\frac{\partial e_{2}}{\partial \nu}=0 & \text { on } \Gamma, \\ e_{1}=e_{2}, \quad \frac{\partial e_{1}}{\partial \nu}=\frac{\partial e_{2}}{\partial \nu} & \text { on } \Gamma_{1}, \\ a_{1} \Delta e_{1}=a_{2} \Delta e_{2}, \quad a_{1} \frac{\partial \Delta e_{1}}{\partial \nu}=a_{2} \frac{\partial \Delta e_{2}}{\partial \nu} & \text { on } \Gamma_{1}\end{cases}
$$

and set

$$
w=\int_{0}^{t} u(s) d s+e, \quad F=\int_{0}^{t} f(s, x) d s+q e-\int_{0}^{t} q(x, s) u(s) d s+q \int_{0}^{t} u(s) d s,
$$


where $u$ is the solution of (1.1). It is easily verified that

$$
\begin{cases}w^{\prime \prime}+a(x) \Delta^{2} w+q w=F & \text { in } Q \\ w(0)=e, \quad w^{\prime}(0)=u^{0} & \text { in } \Omega, \\ w_{2}=\frac{\partial w_{2}}{\partial \nu}=0 & \text { on } \Sigma \\ w_{1}=w_{2}, \quad \frac{\partial w_{1}}{\partial \nu}=\frac{\partial w_{2}}{\partial \nu} & \text { on } \Sigma_{1} \\ a_{1} \Delta w_{1}=a_{2} \Delta w_{2}, \quad a_{1} \frac{\partial \Delta w_{1}}{\partial \nu}=a_{2} \frac{\partial \Delta w_{2}}{\partial \nu} & \text { on } \Sigma_{1} .\end{cases}
$$

Since $\left(e, u^{0}, F\right) \in H_{0}^{2}(\Omega) \times L^{2}(\Omega) \times L^{1}\left(0, T ; L^{2}(\Omega)\right)$, it follows from Lemma 2.2 that there exists a constant $c>0$ such that

$$
\begin{aligned}
\int_{\Sigma}\left|\Delta w_{2}\right|^{2} d \Sigma & \leq c\left[\|e\|_{2, \Omega}^{2}+\left\|u^{0}\right\|_{0, \Omega}^{2}+\|F\|_{L^{1}\left(0, T ; L^{2}(\Omega)\right)}^{2}\right] \\
& \leq c\left[\left\|u^{1}\right\|_{-2, \Omega}^{2}+\left\|u^{0}\right\|_{0, \Omega}^{2}+\|f\|_{L^{2}\left(0, T ; L^{2}(\Omega)\right)}^{2}\right] .
\end{aligned}
$$

Here we have used the fact that $a(x) \Delta^{2}$ is an isomorphism from $H_{0}^{2}(\Omega)$ onto $H^{-2}(\Omega)$. Because $\frac{\partial}{\partial t}$ is an isomorphism from $L^{2}(\Sigma)$ onto $H^{-1}\left(0, T ; L^{2}(\Gamma)\right)$, we conclude that

$$
\Delta u_{2}=\frac{\partial}{\partial t}\left(\Delta w_{2}\right) \in H^{-1}\left(0, T ; L^{2}(\Gamma)\right)
$$

and

$$
\left\|\Delta u_{2}\right\|_{H^{-1}\left(0, T ; L^{2}(\Gamma)\right)}^{2} \leq c\left[\left\|u^{1}\right\|_{-2, \Omega}^{2}+\left\|u^{0}\right\|_{0, \Omega}^{2}+\|f\|_{L^{2}\left(0, T ; L^{2}(\Omega)\right)}^{2}\right] .
$$

4. Observability inequality. The objective of this section is to establish a priori estimates (the observability inequalities), which will permit us to obtain a uniqueness theorem and, a fortiori, theorems of exact controllability. It will also give supplementary information on the space of controllable initial states.

We define the energy of the solution $u$ of (1.1) by

$$
E(u, t)=\frac{1}{2} \int_{\Omega}\left[\left|u^{\prime}(x, t)\right|^{2}+a(x)|\Delta u|^{2}\right] d x .
$$

Then,

$$
E(u, t)=E(u, 0)+\int_{0}^{t} \int_{\Omega}(f-q u) u^{\prime} d x d t
$$

Let $\lambda_{1}$ be the smallest eigenvalue of the operator $\Delta^{2}$ with Dirichlet homogeneous boundary conditions on $L^{2}(\Omega)$ (see (1.6)). We introduce two constants

$$
\begin{aligned}
\mu_{1} & = \begin{cases}\max \left\{\lambda_{1}^{-1}, \lambda_{1}^{-1 / 2}\right\}, & n=1 \\
\lambda_{1}^{-1 / 2}, & n \geq 2\end{cases} \\
R_{*}^{2} & = \begin{cases}\max \left\{R^{2}\left(x^{0}\right), \frac{3}{4}\right\}, & n=1 \\
R^{2}\left(x^{0}\right), & n \geq 2\end{cases}
\end{aligned}
$$


LEMma 4.1 (The observability inequality). Suppose that the boundary $\Gamma$ of $\Omega$ is of class $C^{3}$. Suppose that there exists $x^{0} \in \Omega_{1}$ such that $m(x) \cdot \nu(x) \geq 0$ on $\Gamma_{1}$, where $\nu$ directs towards the exterior of $\Omega_{1}$. Assume $a_{2} \leq a_{1}$ and $T>T\left(x_{0}\right)=\frac{R_{*} \mu_{1}}{\sqrt{a_{2}}}$ and

$$
q_{0}=\max _{(x . t) \in Q}|q(x, t)|<\frac{2\left(T-R_{*} \mu_{1} a_{2}^{-1 / 2}\right)}{T\left[R_{*} \lambda_{1}^{-1 / 2} a_{2}^{-1}\left(3 \mu_{1}+\lambda_{1}^{-1 / 4}\right)+|n-2| \lambda_{1}^{-1} a_{2}^{-1}\right]} .
$$

If $u$ is a weak solution of (1.1) with $f=0$, then

$$
c E(u, 0) \leq \frac{a_{2} R\left(x^{0}\right)}{2} \int_{\Sigma\left(x^{0}\right)}\left|\Delta u_{2}\right|^{2} d \Sigma
$$

where

$$
c=\frac{T\left[2-q_{0}|n-2| \lambda_{1}^{-1} a_{2}^{-1}-q_{0} R_{*} \lambda_{1}^{-1 / 2} a_{2}^{-1}\left(\mu_{1}+\lambda_{1}^{-1 / 4}\right)\right]}{1+q_{0} T \lambda_{1}^{-1 / 2} a_{2}^{-1 / 2}}-2 R_{*} \mu_{1} a_{2}^{-1 / 2} .
$$

Proof. It suffices to prove (4.6) in the case of strong solutions, that is, we assume initial conditions $\left(u^{0}, u^{1}\right) \in H^{4}\left(\Omega, \Gamma_{1}\right) \times H_{0}^{2}(\Omega)$ because we can pass to the limit in the case of weak solutions.

Taking $\rho_{k}=m_{k}$ in Lemma 2.1 , we obtain

$$
\begin{aligned}
& \frac{1}{2} \int_{\Sigma} a_{2} m_{k} \nu_{k}\left|\Delta u_{2}\right|^{2} d \Sigma \\
& =\left.\left(u^{\prime}(t), m_{k} \frac{\partial u(t)}{\partial x_{k}}\right)\right|_{0} ^{T}+2 \int_{0}^{T} E(t) d t \\
& \quad+\frac{n-2}{2} \int_{Q}\left(\left|u^{\prime}\right|^{2}-a(x)|\nabla u|^{2}\right) d x d t+\int_{Q} q m_{k} u \frac{\partial u}{\partial x_{k}} d x d t \\
& \quad+\int_{\Sigma_{1}} a_{1} \Delta u_{1} m_{k} \frac{\partial^{2}}{\partial \nu \partial x_{k}}\left(u_{2}-u_{1}\right) d \Sigma+\frac{1}{2} \int_{\Sigma_{1}} m_{k} \nu_{k}\left(a_{1}\left|\Delta u_{1}\right|^{2}-a_{2}\left|\Delta u_{2}\right|^{2}\right) d \Sigma .
\end{aligned}
$$

On the other hand, multiplying the first equation of (1.1) by $u$ and integrating over $Q$, we obtain

$$
\begin{aligned}
& \left.\left(u^{\prime}, u\right)\right|_{0} ^{T}-\int_{Q}\left|u^{\prime}\right|^{2}+\int_{\Sigma_{1}} a_{1} \frac{\partial \Delta u_{1}}{\partial \nu} u_{1} d \Sigma-\int_{\Sigma_{1}} a_{1} \Delta u_{1} \frac{\partial u_{1}}{\partial \nu} d \Sigma+\int_{Q_{1}} a_{1}\left|\Delta u_{1}\right|^{2} d x d t \\
& -\int_{\Sigma_{1}} a_{2} \frac{\partial \Delta u_{2}}{\partial \nu} u_{2} d \Sigma+\int_{\Sigma_{1}} a_{2} \Delta u_{2} \frac{\partial u_{2}}{\partial \nu} d \Sigma+\int_{Q_{2}} a_{2}\left|\Delta u_{2}\right|^{2} d x d t+\int_{Q} q|u|^{2} d x d t=0 .
\end{aligned}
$$

The transmission condition gives

$$
\left.\left(u^{\prime}(t), u(t)\right)\right|_{0} ^{T}+\int_{Q} q|u|^{2} d x d t=\int_{Q}\left(\left|u^{\prime}\right|^{2}-a(x)|\Delta u|^{2}\right) d x d t .
$$


Therefore, (4.8) becomes

$$
\begin{aligned}
& \frac{1}{2} \int_{\Sigma} a_{2} m_{k} \nu_{k}\left|\Delta u_{2}\right|^{2} d \Sigma \\
& =\left.\left(u^{\prime}(t), m_{k} \frac{\partial u(t)}{\partial x_{k}}+\frac{n-2}{2} u(t)\right)\right|_{0} ^{T}+2 \int_{0}^{T} E(t) d t \\
& \quad+\frac{n-2}{2} \int_{Q} q|u|^{2} d x d t+\int_{Q} q m_{k} u \frac{\partial u}{\partial x_{k}} d x d t \\
& \quad+\int_{\Sigma_{1}} a_{1} \Delta u_{1} m_{k} \frac{\partial^{2}}{\partial \nu \partial x_{k}}\left(u_{2}-u_{1}\right) d \Sigma+\frac{1}{2} \int_{\Sigma_{1}} m_{k} \nu_{k}\left(a_{1}\left|\Delta u_{1}\right|^{2}-a_{2}\left|\Delta u_{2}\right|^{2}\right) d \Sigma
\end{aligned}
$$

We now estimate the right-hand side of (4.10). First, by the Cauchy-Schwartz inequality we have

$$
\begin{aligned}
\left|\left(u^{\prime}(t), m_{k} \frac{\partial u(t)}{\partial x_{k}}+\frac{n-2}{2} u(t)\right)\right| \leq & \frac{R_{*} \mu_{1}}{2 \sqrt{a_{2}}} \int_{\Omega}\left|u^{\prime}(t)\right|^{2} d x \\
& +\frac{a_{2}}{2 R_{*} \sqrt{a_{2}} \mu_{1}} \int_{\Omega}\left|m_{k} \frac{\partial u(t)}{\partial x_{k}}+\frac{n-1}{2} u(t)\right|^{2} d x .
\end{aligned}
$$

Moreover,

$$
\begin{aligned}
\int_{\Omega}\left|m_{k} \frac{\partial u(t)}{\partial x_{k}}+\frac{n-2}{2} u(t)\right|^{2} d x= & \int_{\Omega}\left|m_{k} \frac{\partial u}{\partial x_{k}}\right|^{2} d x+\frac{(n-2)^{2}}{4} \int_{\Omega}|u(t)|^{2} d x \\
& +(n-2)\left(m_{k} \frac{\partial u}{\partial x_{k}}, u(t)\right) .
\end{aligned}
$$

Since

$$
\begin{aligned}
\left(m_{k} \frac{\partial u}{\partial x_{k}}, u(t)\right)= & \frac{1}{2} \int_{\Omega} m_{k} \frac{\partial}{\partial x_{k}}\left(|u(t)|^{2}\right) d x \\
= & \frac{1}{2} \int_{\Gamma_{1}} m_{k} \nu_{k}\left|u_{1}(t)\right|^{2} d \Gamma-\frac{n}{2} \int_{\Omega_{1}}\left|u_{1}(t)\right|^{2} d x \\
& -\frac{1}{2} \int_{\Gamma_{1}} m_{k} \nu_{k}\left|u_{2}(t)\right|^{2} d \Gamma-\frac{n}{2} \int_{\Omega_{2}}\left|u_{2}(t)\right|^{2} d x \\
= & -\frac{n}{2} \int_{\Omega}|u(t)|^{2} d x,
\end{aligned}
$$

then by (1.7) and (1.9) we deduce that

$$
\begin{aligned}
\int_{\Omega}\left|m_{k} \frac{\partial u(t)}{\partial x_{k}}+\frac{n-2}{2} u(t)\right|^{2} d x & =\int_{\Omega}\left|m_{k} \frac{\partial u(t)}{\partial x_{k}}\right|^{2} d x+\frac{2^{2}-n^{2}}{4} \int_{\Omega}|u(t)|^{2} d x \\
& \leq R^{2}\left(x^{0}\right) \int_{\Omega}|\nabla u(t)|^{2} d x+\frac{2^{2}-n^{2}}{4} \int_{\Omega}|u(t)|^{2} d x \\
& \leq R_{*}^{2} \mu_{1}^{2} \int_{\Omega}|\Delta u(t)|^{2} d x
\end{aligned}
$$


Thus, (4.11) becomes

$$
\left|\left(u^{\prime}(t), m_{k} \frac{\partial u(t)}{\partial x_{k}}+\frac{n-2}{2} u(t)\right)\right| \leq \frac{R_{*} \mu_{1}}{\sqrt{a_{2}}} E(u, t), \quad \forall t \in[0, T] .
$$

We then estimate the last two terms of (4.10). Since

$$
\begin{gathered}
\frac{\partial}{\partial x_{k}}\left(\frac{\partial u_{1}}{\partial \nu}\right)=\nu_{k} \frac{\partial^{2} u_{1}}{\partial \nu^{2}}+\sigma_{k}\left(\frac{\partial u_{1}}{\partial \nu}\right), \quad \frac{\partial}{\partial x_{k}}\left(\frac{\partial u_{2}}{\partial \nu}\right)=\nu_{k} \frac{\partial^{2} u_{2}}{\partial \nu^{2}}+\sigma_{k}\left(\frac{\partial u_{2}}{\partial \nu}\right) \text { on } \Sigma_{1} \\
\sigma_{k}\left(\frac{\partial u_{1}}{\partial \nu}\right)=\sigma_{k}\left(\frac{\partial u_{2}}{\partial \nu}\right), \quad \frac{\partial^{2}}{\partial x_{k}^{2}}\left(u_{2}-u_{1}\right)=\nu_{k}^{2} \frac{\partial^{2}}{\partial \nu^{2}}\left(u_{2}-u_{1}\right) \text { on } \Sigma_{1}
\end{gathered}
$$

where $\sigma_{k}$ denote the first-order tangential differential operators on $\Sigma_{1}$ (see [10], Chapter 3 , p. 137), we deduce that

$$
\begin{aligned}
\int_{\Sigma_{1}} a_{1} & \Delta u_{1} m_{k} \frac{\partial^{2}}{\partial \nu \partial x_{k}}\left(u_{2}-u_{1}\right) d \Sigma+\frac{1}{2} \int_{\Sigma_{1}} m_{k} \nu_{k}\left(a_{1}\left|\Delta u_{1}\right|^{2}-a_{2}\left|\Delta u_{2}\right|^{2}\right) d \Sigma \\
& =\int_{\Sigma_{1}} a_{1} \Delta u_{1} m \cdot \nu\left(\frac{\partial^{2} u_{2}}{\partial \nu^{2}}-\frac{\partial^{2} u_{1}}{\partial \nu^{2}}\right) d \Sigma+\frac{1}{2} \int_{\Sigma_{1}} m_{k} \nu_{k}\left(a_{1}\left|\Delta u_{1}\right|^{2}-a_{2}\left|\Delta u_{2}\right|^{2}\right) d \Sigma \\
& =\int_{\Sigma_{1}} a_{1} \Delta u_{1} m \cdot \nu\left(\Delta u_{2}-\Delta u_{1}\right) d \Sigma+\frac{1}{2} \int_{\Sigma_{1}} m_{k} \nu_{k}\left(a_{1}\left|\Delta u_{1}\right|^{2}-a_{2}\left|\Delta u_{2}\right|^{2}\right) d \Sigma \\
& =\frac{a_{1}\left(a_{1}-a_{2}\right)}{2 a_{2}} \int_{\Sigma_{1}} m \cdot \nu\left|\Delta u_{1}\right|^{2} d \Sigma \\
& \geq 0
\end{aligned}
$$

since $a_{1} \geq a_{2}$ and $m \cdot \nu \geq 0$ on $\Gamma_{1}$. It therefore follows from (4.2), (4.10), (4.15), and (4.16) that

$$
\begin{aligned}
& \frac{1}{2} \int_{\Sigma} a_{2} m_{k} \nu_{k}\left|\Delta u_{2}\right|^{2} d \Sigma \\
& \geq 2 \int_{0}^{T} E(u, t) d t-\frac{R_{*} \mu_{1}}{\sqrt{a_{2}}}[E(u, 0)+E(u, T)] \\
& \quad-\frac{q_{0}|n-2|}{\lambda_{1} a_{2}} \int_{0}^{T} E(u, t) d t-\frac{q_{0} R_{*}}{\lambda_{1}^{3 / 4} a_{2}} \int_{0}^{T} E(u, t) d t \\
&=\left(2-q_{0}|n-2| \lambda_{1}^{-1} a_{2}^{-1}-q_{0} R_{*} \lambda_{1}^{-3 / 4} a_{2}^{-1}\right) \int_{0}^{T} E(u, t) d t \\
&-2 R_{*} \mu_{1} a_{2}^{-1 / 2} E(u, 0)+R_{*} \mu_{1} a_{2}^{-1 / 2} \int_{0}^{T} \int_{\Omega} q u u^{\prime} d x d t \\
& \geq {\left[2-q_{0}|n-2| \lambda_{1}^{-1} a_{2}^{-1}-q_{0} R_{*} \lambda_{1}^{-1 / 2} a_{2}^{-1}\left(\mu_{1}+\lambda_{1}^{-1 / 4}\right)\right] \int_{0}^{T} E(u, t) d t } \\
&-2 R_{*} \mu_{1} a_{2}^{-1 / 2} E(u, 0) .
\end{aligned}
$$


Moreover, by (4.2) we have

$$
\begin{aligned}
\int_{0}^{T} E(u, t) d t & =T E(u, 0)-\int_{0}^{T} \int_{0}^{t} \int_{\Omega} q u(x, s) u^{\prime}(x, s) d x d s d t \\
& \geq T E(u, 0)-q_{0} T \lambda_{1}^{-1 / 2} a_{2}^{-1 / 2} \int_{0}^{T} E(u, t) d t
\end{aligned}
$$

which implies

$$
\int_{0}^{T} E(u, t) d t \geq \frac{T}{1+q_{0} T \lambda_{1}^{-1 / 2} a_{2}^{-1 / 2}} E(u, 0) .
$$

Combining (4.5), (4.17), and (4.18) gives (4.6).

In order to relax the restrictions on $T$ and $q(x, t)$, we need the following unique theorems.

ThEOREM 4.2. Suppose there exists $x^{0} \in \Omega_{1}$ such that $m(x) \cdot \nu(x) \geq 0$ on $\Gamma_{1}$, where $\nu$ directs towards the exterior of $\Omega_{1}$. Assume $a_{2} \leq a_{1}$. Let $q \in W^{1, \infty}(\Omega)$ be such that $m(x) \cdot \nabla q \leq 0$ on $\Omega$. If

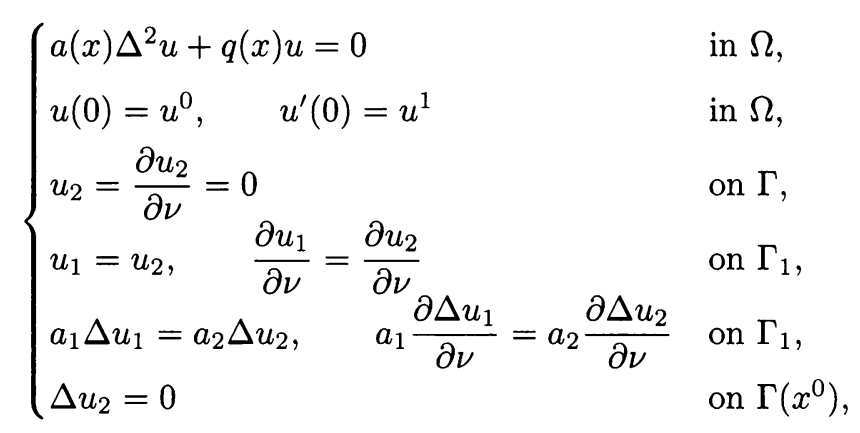

then

$$
u=0 \text { in } \Omega \text {. }
$$

Proof. Multiplying (4.19) by $m_{k} \frac{\partial u}{\partial x_{k}}$ and integrating over $\Omega$, we obtain

$$
\int_{\Omega} a(x) m_{k} \frac{\partial u}{\partial x_{k}} \Delta^{2} u d x+\int_{\Omega} q m_{k} \frac{\partial u}{\partial x_{k}} u d x=0 .
$$

It follows from (2.6) that

$$
\begin{aligned}
\int_{\Omega} a(x) m_{k} \frac{\partial u}{\partial x_{k}} \Delta^{2} u d x= & -\frac{1}{2} \int_{\Gamma} a_{2} m_{k} \nu_{k}\left|\Delta u_{2}\right|^{2} d \Gamma+\left(2-\frac{n}{2}\right) \int_{\Omega} a(x)|\Delta u|^{2} d x \\
& +\int_{\Gamma_{1}} a_{1} \Delta u_{1} m_{k} \frac{\partial^{2}}{\partial \nu \partial x_{k}}\left(u_{2}-u_{1}\right) d \Gamma \\
& +\frac{1}{2} \int_{\Gamma_{1}} m_{k} \nu_{k}\left(a_{1}\left|\Delta u_{1}\right|^{2}-a_{2}\left|\Delta u_{2}\right|^{2}\right) d \Gamma
\end{aligned}
$$

Moreover, we have

$$
\int_{\Omega} q m_{k} \frac{\partial u}{\partial x_{k}} u d x=-\frac{n}{2} \int_{\Omega} q|u|^{2} d x-\frac{1}{2} \int_{\Omega} m_{k} \frac{\partial q}{\partial x_{k}}|u|^{2} u d x .
$$


On the other hand, multiplying (4.19) by $u$ and integrating over $\Omega$, we obtain

$$
\int_{\Omega}\left(a(x)|\Delta u|^{2}+q|u|^{2}\right) d x=0 .
$$

We then deduce from (4.21)-(4.24) that

$$
\begin{aligned}
0= & -\frac{1}{2} \int_{\Gamma_{*}\left(x^{0}\right)} a_{2} m_{k} \nu_{k}\left|\Delta u_{2}\right|^{2} d \Gamma+2 \int_{\Omega} a(x)|\Delta u|^{2} d x-\frac{1}{2} \int_{\Omega} m_{k} \frac{\partial q}{\partial x_{k}}|u|^{2} u d x \\
& +\int_{\Gamma_{1}} a_{1} \Delta u_{1} m_{k} \frac{\partial^{2}}{\partial \nu \partial x_{k}}\left(u_{2}-u_{1}\right) d \Gamma+\frac{1}{2} \int_{\Gamma_{1}} m_{k} \nu_{k}\left(a_{1}\left|\Delta u_{1}\right|^{2}-a_{2}\left|\Delta u_{2}\right|^{2}\right) d \Gamma,
\end{aligned}
$$

which, by (4.16), implies (4.20) since $m \cdot \nu \leq 0$ on $\Gamma_{*}\left(x^{0}\right)$ and $m(x) \cdot \nabla q \leq 0$ on $\Omega$.

If $-q$ is a constant and not an eigenvalue of $a(x) \Delta^{2}$, then the condition " $\Delta u_{2}=0$ on $\Gamma\left(x^{0}\right)$ " is not required. In addition, there are functions $q$ that satisfy the condition of Theorem 4.2, for example, $q \equiv c$ (a constant) and $q=-\left|x-x^{0}\right|^{2}$.

The following uniqueness theorem is an extension of a theorem of Zuazua (see [10], Appendix 1) to the case of transmission with lower-order terms.

Theorem 4.3. Suppose the boundary $\Gamma$ of $\Omega$ is of class $C^{3}$. Suppose there exists $x^{0} \in \Omega_{1}$ such that $m(x) \cdot \nu(x) \geq 0$ on $\Gamma_{1}$, where $\nu$ directs towards the exterior of $\Omega_{1}$. Assume $a_{2} \leq a_{1}$ and $T>0$. Let either $q \in L^{\infty}\left(0, T ; W^{1, \infty}(\Omega)\right)$ be such that $m(x) \cdot \nabla q\left(x, t_{0}\right) \leq 0$ on $\Omega$ for some $t_{0} \in[0, T]$ or $q \in L^{\infty}(Q)$ be such that

$$
q_{0}<\frac{2}{R_{*} \lambda_{1}^{-1 / 2} a_{2}^{-1}\left(3 \mu_{1}+\lambda_{1}^{-1 / 4}\right)+|n-2| \lambda_{1}^{-1} a_{2}^{-1}} .
$$

If

$$
u \in X=C\left([0, T] ; H_{0}^{2}(\Omega)\right) \cap C^{1}\left([0, T] ; L^{2}(\Omega)\right)
$$

is a solution of

$$
\begin{cases}u^{\prime \prime}+a(x) \Delta^{2} u+q u=0 & \text { in } Q, \\ u_{2}=\frac{\partial u_{2}}{\partial \nu}=0 & \text { on } \Sigma, \\ u_{1}=u_{2}, \quad \frac{\partial u_{1}}{\partial \nu}=\frac{\partial u_{2}}{\partial \nu} & \text { on } \Sigma_{1}, \\ a_{1} \Delta u_{1}=a_{2} \Delta u_{2}, \quad a_{1} \frac{\partial \Delta u_{1}}{\partial \nu}=a_{2} \frac{\partial \Delta u_{2}}{\partial \nu} & \text { on } \Sigma_{1}\end{cases}
$$

such that

$$
\Delta u_{2}=0 \quad \text { on } \Sigma\left(x^{0}\right),
$$

then

$$
u=0 \text { in } Q
$$

Proof. Set

$$
Y=\{u \in X: u \text { satisfies (4.27), (4.28)\} }
$$

with the norm induced by $X$. It suffices to prove $Y=\{0\}$. 
Because by (4.2) we have

$$
\begin{aligned}
\int_{0}^{T} E(u, t) d t & =T E(u, 0)-\int_{0}^{T} \int_{0}^{t} \int_{\Omega} q u u^{\prime} d x d s d t \\
& =T E(u, 0)-\frac{1}{2} \int_{0}^{T} \int_{\Omega} q\left(u^{2}(x, t)-u^{2}(x, 0)\right) d x d t
\end{aligned}
$$

we deduce from (1.12) and (4.10) that

$$
\begin{aligned}
E(u, 0) & \leq c_{1}\left\|\Delta u_{2}\right\|_{L^{2}\left(\Sigma\left(x^{0}\right)\right)}^{2}+c_{2}\|u\|_{L^{\infty}\left(0, T ; H_{0}^{1}(\Omega)\right)}^{2}+\left|\left(u^{\prime}(t), m_{k} \frac{\partial u(t)}{\partial x_{k}}+\frac{n-2}{2} u(t)\right)\right|_{0}^{T} \mid \\
& \leq c_{1}\left\|\Delta u_{2}\right\|_{L^{2}\left(\Sigma\left(x^{0}\right)\right)}^{2}+c(\varepsilon)\|u\|_{L^{\infty}\left(0, T ; H_{0}^{1}(\Omega)\right)}^{2}+\varepsilon E(u, 0),
\end{aligned}
$$

which implies

$$
E(u, 0) \leq c\left[\left\|\Delta u_{2}\right\|_{L^{2}\left(\Sigma\left(x^{0}\right)\right)}^{2}+\|u\|_{L^{\infty}\left(0, T ; H_{0}^{1}(\Omega)\right)}^{2}\right],
$$

for $u \in X$ satisfying (4.27).

On the other hand, we show there exists a constant $c>0$ such that

$$
\|u\|_{L^{\infty}\left(0, T ; H_{0}^{1}(\Omega)\right)}^{2} \leq c\left[\left\|\Delta u_{2}\right\|_{L^{2}\left(\Sigma\left(x^{0}\right)\right)}^{2}+\|u\|_{L^{\infty}\left(0, T^{\prime} ; L^{2}(\Omega)\right)}^{2}\right]
$$

for $u \in X$ satisfying (4.27). In fact, if (4.30) is not true, there exists a sequence $\left\{u_{n}\right\}$ of solutions of (4.27) with (4.26) such that

$$
\left\|\Delta u_{n 2}\right\|_{L^{2}\left(\Sigma\left(x^{0}\right)\right)}^{2}+\left\|u_{n}\right\|_{L^{\infty}\left(0, T ; L^{2}(\Omega)\right)}^{2} \rightarrow 0 \quad(n \rightarrow \infty)
$$

and

$$
\left\|u_{n}\right\|_{L^{\infty}\left(0, T ; H_{0}^{1}(\Omega)\right)}=1 .
$$

It therefore follows from (4.29) and (1.12) that $\left\{u_{n}\right\}$ is bounded in $X$, and then relatively compact in $L^{\infty}\left(0, T ; H_{0}^{1}(\Omega)\right)$ because the injection: $X \rightarrow L^{\infty}\left(0, T ; H_{0}^{1}(\Omega)\right)$ is compact due to Simon's results [13]. By extracting a subsequence, we may as well assume $\left\{u_{n}\right\}$ converges strongly to $u$ in $L^{\infty}\left(0, T ; H_{0}^{1}(\Omega)\right)$. Thus by (4.32) we obtain

$$
\|u\|_{L^{\infty}\left(0, T ; H_{0}^{1}(\Omega)\right)}=1 .
$$

However, (4.31) implies

$$
u=0 \text { in } Q .
$$

This is in contradiction with (4.33).

By (4.29) and (4.30) we have

$$
E(u, 0) \leq c\left[\left\|\Delta u_{2}\right\|_{L^{2}\left(\Sigma\left(x^{0}\right)\right)}^{2}+\|u\|_{L^{\infty}\left(0, T ; L^{2}(\Omega)\right)}^{2}\right]
$$

for $u \in X$ satisfying (4.27). By the argument of density, (4.34) still holds for $u \in$ $L^{\infty}\left(0, T ; L^{2}(\Omega)\right)$ satisfying $(4.27)$.

We observe that if $u \in Y$ then $v=u^{\prime}$ satisfies (4.27) and (4.28), and $v \in L^{\infty}(0, T$; $\left.L^{2}(\Omega)\right)$. Hence by $(4.34)$ we deduce that

$$
\left(v(0), v^{\prime}(0)\right) \in H_{0}^{2}(\Omega) \times L^{2}(\Omega),
$$

and then by (1.12) we deduce that $v \in X$. It therefore follows that

$$
u \rightarrow u^{\prime} \text { is a continuous operator from } Y \text { to } Y,
$$


since by (4.34) $\frac{d}{d t}: Y \rightarrow Y$ maps a bounded subset of $Y$ into a bounded subset of $Y$. Moreover, the injection $\left\{u \in Y: u^{\prime} \in Y\right\} \rightarrow Y$ is compact. Thus we deduce that the dimension of $Y$ is finite.

Suppose $Y \neq\{0\}$. Then by complexifying $Y$, it follows from (4.35) that there exists $\lambda \in \mathbf{C}$ and $u \in Y-\{0\}$ such that

$$
u^{\prime}=\lambda u
$$

This implies

$$
u(x, t)=e^{\lambda t} u(x, 0) .
$$

Since

$$
\Delta u_{2}(x, 0)=0 \quad \text { on } \Gamma\left(x^{0}\right)
$$

it follows from (4.36) that

$$
\Delta u_{2}(x, t)=0 \quad \text { on } \Gamma\left(x^{0}\right) \times(-\infty,+\infty) .
$$

On the other hand, if $q \in L^{\infty}(Q)$ such that (4.25) holds, then we can find $T_{0}>R_{*} \mu_{1} a_{2}^{-1 / 2}$ such that

$$
q_{0}<\frac{2\left(T_{0}-R_{*} \mu_{1} a_{2}^{-1 / 2}\right)}{T_{0}\left[R_{*} \lambda_{1}^{-1 / 2} a_{2}^{-1}\left(3 \mu_{1}+\lambda_{1}^{-1 / 4}\right)+|n-2| \lambda_{1}^{-1} a_{2}^{-1}\right]} .
$$

Then by Lemma 4.1 we deduce that

$$
u=0 \text { in } Q \text {. }
$$

If $q \in L^{\infty}\left(0, T ; W^{1, \infty}(\Omega)\right)$ satisfies $m(x) \cdot \nabla q\left(x, t_{0}\right) \leq 0$ on $\Omega$ for some $t_{0} \in[0, T]$, then we substitute (4.36) into (4.27) and we obtain

$$
\begin{cases}a(x) \Delta^{2} u(x, 0)+\left(q\left(x, t_{0}\right)+\lambda^{2}\right) u(x, 0)=0 & \text { in } \Omega, \\ u_{2}(x, 0)=\frac{\partial u_{2}(x, 0)}{\partial \nu}=0 & \text { on } \Gamma, \\ u_{1}(x, 0)=u_{2}(x, 0), \quad \frac{\partial u_{1}(x, 0)}{\partial \nu}=\frac{\partial u_{2}(x, 0)}{\partial \nu} & \text { on } \Gamma_{1}, \\ a_{1} \Delta u_{1}(x, 0)=a_{2} \Delta u_{2}(x, 0), \quad a_{1} \frac{\partial \Delta u_{1}(x, 0)}{\partial \nu}=a_{2} \frac{\partial \Delta u_{2}(x, 0)}{\partial \nu} & \text { on } \Gamma_{1}, \\ \Delta u_{2}(x, 0)=0 & \text { on } \Gamma\left(x^{0}\right) .\end{cases}
$$

By Lemma 4.2, we also have (4.39). This is in contradiction with $u \in Y-\{0\}$.

Using Theorem 4.3 , we prove

LEMMA 4.4 (The observability inequality). Suppose the boundary $\Gamma$ of $\Omega$ is of class $C^{3}$ and the boundary $\Gamma_{1}$ of $\Omega_{1}$ is of class $C^{4}$. Suppose there exists $x^{0} \in \Omega_{1}$ such that $m(x) \cdot \nu(x) \geq 0$ on $\Gamma_{1}$, where $\nu$ directs towards the exterior of $\Omega_{1}$. Assume $a_{2} \leq a_{1}$ and $T>0$. Let $q \in L^{\infty}\left(0, T ; W^{1, \infty}(\Omega)\right)$ be such that $m(x) \cdot \nabla q\left(x, t_{0}\right) \leq 0$ on $\Omega$ for some $t_{0} \in[0, T]$ or $q \in L^{\infty}(Q)$ be such that (4.25) holds. Then there is $c>0$ such that for all weak solutions $u$ of (1.1) with $f=0$ we have

$$
E(0) \leq c \int_{\Sigma\left(x^{0}\right)}\left|\Delta u_{2}\right|^{2} d \Sigma .
$$


Proof. By (4.29), there exists a constant $c>0$ such that

$$
E(u, 0) \leq c\left[\left\|\Delta u_{2}\right\|_{L^{2}\left(\Sigma\left(x^{0}\right)\right)}^{2}+\|u\|_{L^{\infty}\left(0, T ; H_{0}^{1}(\Omega)\right)}^{2}\right]
$$

for solutions $u$ of (1.1) with $f=0$.

Furthermore, we show there exists a constant $c>0$ such that

$$
\|u\|_{L^{\infty}\left(0, T ; H_{0}^{1}(\Omega)\right)} \leq c\left\|\Delta u_{2}\right\|_{L^{2}\left(\Sigma\left(x^{0}\right)\right)}
$$

for solutions $u$ of (1.1) with $f=0$. In fact, if (4.42) is not true, there exists a sequence $\left\{u_{n}\right\}$ of solutions of (1.1) with $f=0$ such that

$$
\left\|\Delta u_{n 2}\right\|_{L^{2}\left(\Sigma\left(x^{0}\right)\right)} \rightarrow 0 \quad(n \rightarrow \infty)
$$

and

$$
\left\|u_{n}\right\|_{L^{\infty}\left(0, T ; H_{0}^{1}(\Omega)\right)}=1 .
$$

It therefore follows from (4.41) and (1.12) that $\left\{u_{n}\right\}$ is bounded in $C\left([0, T] ; H_{0}^{2}(\Omega)\right) \cap$ $C^{1}\left([0, T] ; L^{2}(\Omega)\right)$, and then relatively compact in $L^{\infty}\left(0, T ; H_{0}^{1}(\Omega)\right)$ because the injection: $C\left([0, T] ; H_{0}^{2}(\Omega)\right) \cap C^{1}\left([0, T] ; L^{2}(\Omega)\right) \rightarrow L^{\infty}\left(0, T ; H_{0}^{1}(\Omega)\right)$ is compact due to Simon's results [13]. By extracting a subsequence, we may as well assume $\left\{u_{n}\right\}$ converges strongly to $u$ in $L^{\infty}\left(0, T ; H_{0}^{1}(\Omega)\right)$. Thus by $(4.44)$ we obtain

$$
\|u\|_{L^{\infty}\left(0, T ; H_{0}^{1}(\Omega)\right)}=1 .
$$

In addition, $\left\{u_{n}\right\}$ and $\left\{u_{n}^{\prime}\right\}$ converge to $u$ star-weakly in $L^{\infty}\left(0, T ; H_{0}^{2}(\Omega)\right)$ and $L^{\infty}(0, T$; $\left.L^{2}(\Omega)\right)$ respectively. Thus $u$ is a solution of (1.1) with

$$
C\left([0, T] ; H_{0}^{2}(\Omega)\right) \cap C^{1}\left([0, T] ; L^{2}(\Omega)\right) .
$$

By (4.41), we have

$$
E\left(u_{n}-u_{m}, 0\right) \leq c\left[\left\|\Delta u_{n 2}-\Delta u_{m 2}\right\|_{L^{2}\left(\Sigma\left(x^{0}\right)\right)}^{2}+\left\|u_{n}-u_{m}\right\|_{L^{\infty}\left(0, T ; H_{0}^{1}(\Omega)\right)}^{2}\right],
$$

which gives

$$
E\left(u_{n}-u, 0\right) \leq c\left[\left\|\Delta u_{n 2}\right\|_{L^{2}\left(\Sigma\left(x^{0}\right)\right)}^{2}+\left\|u_{n}-u\right\|_{L^{\infty}\left(0, T ; H_{0}^{1}(\Omega)\right)}^{2}\right] .
$$

By (2.7), we have

$$
\left\|\Delta u_{n 2}-\Delta u_{2}\right\|_{L^{2}\left(\Sigma\left(x^{0}\right)\right)}^{2} \leq c E\left(u_{n}-u, 0\right) .
$$

It therefore follows from (4.43), (4.46), and (4.47) that

$$
\Delta u_{2}=0 \text { on } \Sigma\left(x^{0}\right) .
$$

By Theorem 4.3 we deduce

$$
u=0 \quad \text { in } Q .
$$

This is in contradiction with (4.45).

Finally, (4.40) follows from (4.41) and (4.42). 
LEMMA 4.5 (The observability inequality). In addition to all assumptions of Lemma 4.4, suppose that the boundary $\Gamma$ of $\Omega$ is of class $C^{4}$ and that $q$ is independent of $t$ with $q \in W^{2, \infty}(\Omega)$. Then there is $c>0$ such that for all strong solutions $u$ of (1.1) with $f=0$ we have

$$
\left\|u^{0}\right\|_{H^{4}\left(\Omega, \Gamma_{1}\right)}^{2}+\left\|u^{1}\right\|_{2, \Omega}^{2} \leq c \int_{\Sigma\left(x^{0}\right)}\left(\left|\Delta u_{2}^{\prime}\right|^{2}+\left|\Delta u_{2}\right|^{2}\right) d \Sigma .
$$

Proof. Set $w=u^{\prime}$. Then $w$ satisfies

$$
\begin{cases}w^{\prime \prime}+a(x) \Delta^{2} w+q w=0 & \text { in } Q \\ w(0)=u^{1}, \quad w^{\prime}(0)=-a(x) \Delta^{2} u^{0}-q u^{0} & \text { in } \Omega \\ w_{2}=\frac{\partial w_{2}}{\partial \nu}=0 & \text { on } \Sigma \\ w_{1}=w_{2}, \quad \frac{\partial w_{1}}{\partial \nu}=\frac{\partial w_{2}}{\partial \nu} & \text { on } \Sigma_{1} \\ a_{1} \Delta w_{1}=a_{2} \Delta w_{2}, \quad a_{1} \frac{\partial \Delta w_{1}}{\partial \nu}=a_{2} \frac{\partial \Delta w_{2}}{\partial \nu} & \text { on } \Sigma_{1} .\end{cases}
$$

It therefore follows from Lemma 4.4 that

$$
\int_{\Sigma\left(x^{0}\right)}\left|\Delta u_{2}^{\prime}\right|^{2} d \Sigma=\int_{\Sigma\left(x^{0}\right)}\left|\Delta w_{2}\right|^{2} d \Sigma \geq c\left[\left\|a \Delta^{2} u^{0}\right\|_{0}^{2}-q_{0}\left\|u^{0}\right\|_{0}^{2}+\left\|u^{1}\right\|_{2, \Omega}^{2}\right]
$$

which, by Lemma 4.4 , gives (4.49).

If $q=0$, then (4.49) becomes

$$
\left\|u^{0}\right\|_{H^{4}\left(\Omega, \Gamma_{1}\right)}^{2}+\left\|u^{1}\right\|_{2, \Omega}^{2} \leq c \int_{\Sigma\left(x^{0}\right)}\left|\Delta u_{2}^{\prime}\right|^{2} d \Sigma .
$$

LEMMA 4.6 (The observability inequality). In addition to all assumptions of Lemma 4.4, suppose the boundary $\Gamma$ of $\Omega$ is of class $C^{4}$ and $q$ is independent of $t$ with $q \in W^{2, \infty}(\Omega)$. Let $q$ be such that $a(x) \Delta^{2}+q$ is an isomorphism from $H_{0}^{2}(\Omega)$ onto $H^{-2}(\Omega)$. Then there is $c>0$ such that for all ultraweak solutions $u$ of (1.1) with $f=0$ we have

$$
\left\|\Delta u_{2}\right\|_{H^{-1}\left(0, T ; L^{2}\left(\Gamma\left(x^{0}\right)\right)\right)} \geq c\left[\left\|u^{1}\right\|_{-2, \Omega}+\left\|u^{0}\right\|_{0, \Omega}\right] .
$$

Proof. Let $e$ be the solution of

$$
\begin{cases}a(x) \Delta^{2} e+q e=-u^{1} & \text { in } \Omega, \\ e_{2}=\frac{\partial e_{2}}{\partial \nu}=0 & \text { on } \Gamma, \\ e_{1}=e_{2}, \quad \frac{\partial e_{1}}{\partial \nu}=\frac{\partial e_{2}}{\partial \nu} & \text { on } \Gamma_{1}, \\ a_{1} \Delta e_{1}=a_{2} \Delta e_{2}, \quad a_{1} \frac{\partial \Delta e_{1}}{\partial \nu}=a_{2} \frac{\partial \Delta e_{2}}{\partial \nu} & \text { on } \Gamma_{1},\end{cases}
$$

and set

$$
w=\int_{0}^{t} u(s) d s+e
$$


where $u$ is the solution of (1.1) with $f=0$. It is easily verified that

$$
\begin{cases}w^{\prime \prime}+a(x) \Delta^{2} w+q w=0 & \text { in } Q, \\ w(0)=e, \quad w^{\prime}(0)=u^{0} & \text { in } \Omega, \\ w_{2}=\frac{\partial w_{2}}{\partial \nu}=0 & \text { on } \Sigma, \\ w_{1}=w_{2}, \quad \frac{\partial w_{1}}{\partial \nu}=\frac{\partial w_{2}}{\partial \nu} & \text { on } \Sigma_{1}, \\ a_{1} \Delta w_{1}=a_{2} \Delta w_{2}, \quad a_{1} \frac{\partial \Delta w_{1}}{\partial \nu}=a_{2} \frac{\partial \Delta w_{2}}{\partial \nu} & \text { on } \Sigma_{1} .\end{cases}
$$

Since $\left(e, u^{0}\right) \in H_{0}^{2}(\Omega) \times L^{2}(\Omega)$, it follows from Lemma 4.4 that there exists a constant $c>0$ such that

$$
\begin{aligned}
\int_{\Sigma}\left|\Delta w_{2}\right|^{2} d \Sigma & \geq c\left[\|e\|_{2, \Omega}^{2}+\left\|u^{0}\right\|_{0, \Omega}^{2}\right] \\
& \geq c\left[\left\|u^{1}\right\|_{-2, \Omega}^{2}+\left\|u^{0}\right\|_{0, \Omega}^{2}\right] .
\end{aligned}
$$

Here we have used the fact that $a(x) \Delta^{2}+q$ is an isomorphism from $H_{0}^{2}(\Omega)$ onto $H^{-2}(\Omega)$. Because $\frac{\partial}{\partial t}$ is an isomorphism from $L^{2}(\Sigma)$ onto $H^{-1}\left(0, T ; L^{2}(\Gamma)\right)$, we conclude

$$
\Delta u_{2}=\frac{\partial}{\partial t}\left(\Delta w_{2}\right) \in H^{-1}\left(0, T ; L^{2}(\Gamma)\right)
$$

and

$$
\left\|\Delta u_{2}\right\|_{H^{-1}\left(0, T ; L^{2}(\Gamma)\right)} \geq c\left[\left\|u^{1}\right\|_{-2, \Omega}+\left\|u^{0}\right\|_{0, \Omega}\right] .
$$

5. Exact controllability. We are now ready to present the main theorems of this paper.

Theorem 5.1. Suppose the boundary $\Gamma$ of $\Omega$ is of class $C^{3}$. Suppose there exists $x^{0} \in \Omega_{1}$ such that $m(x) \cdot \nu(x) \geq 0$ on $\Gamma_{1}$, where $\nu$ directs towards the exterior of $\Omega_{1}$. Assume $a_{2} \leq a_{1}$ and $T>0$. Let either $q \in L^{\infty}\left(0, T ; W^{1, \infty}(\Omega)\right)$ be such that $m(x) \cdot \nabla q\left(x, t_{0}\right) \leq 0$ on $\Omega$ for some $t_{0} \in[0, T]$ or let $q \in L^{\infty}(Q)$ be such that

$$
q_{0}<\frac{2}{R_{*} \lambda_{1}^{-1 / 2} a_{2}^{-1}\left(3 \mu_{1}+\lambda_{1}^{-1 / 4}\right)+|n-2| \lambda_{1}^{-1} a_{2}^{-1}} .
$$

Then for all initial states $\left(y^{0}, y^{1}\right) \in L^{2}(\Omega) \times H^{-2}(\Omega)$, there exists a control

$$
\phi \in L^{2}(\Sigma) \text { with } \phi=0 \text { on } \Sigma_{*}\left(x^{0}\right)
$$

driving system (0.1) to rest. 
Proof. We apply HUM. We first consider the problem:

$$
\begin{cases}u^{\prime \prime}+a(x) \Delta^{2} u+q u=0 & \text { in } Q, \\ u(0)=u^{0}, \quad u^{\prime}(0)=u^{1} & \text { in } \Omega, \\ u_{2}=\frac{\partial u_{2}}{\partial \nu}=0 & \text { on } \Sigma, \\ u_{1}=u_{2}, \quad \frac{\partial u_{1}}{\partial \nu}=\frac{\partial u_{2}}{\partial \nu} & \text { on } \Sigma_{1}, \\ a_{1} \Delta u_{1}=a_{2} \Delta u_{2}, \quad a_{1} \frac{\partial \Delta u_{1}}{\partial \nu}=a_{2} \frac{\partial \Delta u_{2}}{\partial \nu} & \text { on } \Sigma_{1} .\end{cases}
$$

For any $\left(u^{0}, u^{1}\right) \in H_{0}^{2}(\Omega) \times L^{2}(\Omega)$, problem (5.3) has a unique solution $u$ with

$$
\Delta u_{2} \in L^{2}(\Sigma)
$$

because of Theorem 1.4 and Lemma 2.2 .

Using the solution $u$ of (5.3), we then consider the backward problem:

$$
\begin{cases}v^{\prime \prime}+a(x) \Delta^{2} v+q v=0 & \text { in } Q, \\ v(T)=v^{\prime}(T)=0 & \text { in } \Omega, \\ v_{2}=0 & \text { on } \Sigma, \\ \frac{\partial v_{2}}{\partial \nu}= \begin{cases}\Delta u_{2} \quad \text { on } \Sigma\left(x^{0}\right), & \text { on } \Sigma_{*}\left(x^{0}\right), \\ 0 \quad \frac{\partial v_{1}}{\partial \nu}=\frac{\partial v_{2}}{\partial \nu} & \text { on } \Sigma_{1} \\ v_{1}=v_{2}, \quad a_{1} \frac{\partial \Delta v_{1}}{\partial \nu}=a_{2} \frac{\partial \Delta v_{2}}{\partial \nu} & \text { on } \Sigma_{1}\end{cases} \end{cases}
$$

It follows from Theorem 3.2 that problem (5.5) has a unique ultraweak solution $v$ with

$$
v \in C\left([0, T] ; L^{2}(\Omega)\right) \times C^{1}\left([0, T] ; H^{-2}(\Omega)\right) .
$$

We then define a linear operator $\Lambda$ by

$$
\Lambda\left(u^{0}, u^{1}\right)=\left(v^{\prime}(0),-v(0)\right) .
$$

Multiplying the first equation of (5.3) by $v$ and integrating over $Q$, we find

$$
\begin{aligned}
\left\langle\Lambda\left(u^{0}, u^{1}\right),\left(u^{0}, u^{1}\right)\right\rangle & =\left\langle v^{\prime}(0), u^{0}\right\rangle-\int_{\Omega} v(0) u^{1} d x \\
& =\int_{\Sigma\left(x^{0}\right)} a_{2}\left|\Delta u_{2}\right|^{2} d \Sigma .
\end{aligned}
$$

It therefore follows from Lemma 2.2, Lemma 4.4, and the Lax-Milgram Theorem that $\Lambda$ is an isomorphism from $H_{0}^{2}(\Omega) \times L^{2}(\Omega)$ onto $H^{-2}(\Omega) \times L^{2}(\Omega)$. This means that for all $\left(y^{1},-y^{0}\right) \in H^{-2}(\Omega) \times L^{2}(\Omega)$, the equation

$$
\Lambda\left(u^{0}, u^{1}\right)=\left(y^{1},-y^{0}\right)
$$


has a unique solution $\left(u^{0}, u^{1}\right)$. With this initial condition we solve Problem (5.3), and then solve Problem (5.5). Then we have found a control

$$
\phi= \begin{cases}\Delta u_{2} & \text { on } \Sigma\left(x^{0}\right), \\ 0 & \text { on } \Sigma_{*}\left(x^{0}\right),\end{cases}
$$

such that

$$
y(x, t ; \phi)=v(x, t ; \phi)
$$

is the solution of (0.1) satisfying

$$
y(x, T ; \phi)=y^{\prime}(x, T ; \phi)=0 \quad \text { in } \Omega .
$$

This completes the proof.

Based on Lemma 2.3 and Lemma 4.5, we obtain

TheOREM 5.2. Suppose all assumptions of Lemma 4.5 hold. Then for all initial states

$$
\left(y^{0}, y^{1}\right) \in H^{-2}(\Omega) \times\left(H^{4}\left(\Omega, \Gamma_{1}\right)\right)^{\prime},
$$

there exists a control

$$
\phi \in\left(H^{1}\left(0, T ; L^{2}\left(\Gamma\left(x^{0}\right)\right)\right)\right)^{\prime}
$$

driving system (0.1) to rest.

Proof. The proof is the same as the one of Theorem 5.1 except that $H_{0}^{2}(\Omega) \times L^{2}(\Omega)$ is replaced by $H^{4}\left(\Omega, \Gamma_{1}\right) \times H_{0}^{2}(\Omega)$ and $\Delta u_{2}$ in (5.5) is replaced by $-\frac{\partial}{\partial t}\left(\Delta u_{2}^{\prime}\right)+\Delta u_{2}$. At this time, we get a control

$$
\phi= \begin{cases}-\frac{\partial}{\partial t}\left(\Delta u_{2}^{\prime}\right)+\Delta u_{2} & \text { on } \Sigma\left(x^{0}\right), \\ 0 & \text { on } \Sigma_{*}\left(x^{0}\right) .\end{cases}
$$

Based on Lemma 3.8 and Lemma 4.6 , we obtain

Theorem 5.3. Suppose all assumptions of Lemma 4.6 hold. Then for all initial states

$$
\left(y^{0}, y^{1}\right) \in H_{0}^{2}(\Omega) \times L^{2}(\Omega)
$$

there exists a control

$$
\phi \in H_{0}^{1}\left(0, T ; L^{2}\left(\Gamma\left(x^{0}\right)\right)\right)
$$

driving system $(0.1)$ to rest.

Proof. The proof is the same as the one of Theorem 5.1 except that $H_{0}^{2}(\Omega) \times L^{2}(\Omega)$ is replaced by $L^{2}(\Omega) \times H^{-2}(\Omega)$ and $\Delta u_{2}$ in (5.5) is replaced by $\psi$, where $\psi \in H_{0}^{1}(0, T$; $\left.L^{2}\left(\Gamma\left(x^{0}\right)\right)\right)$ is such that

$$
\left\langle\Delta u_{2}, \psi\right\rangle=\left\|\Delta u_{2}\right\|_{H^{-1}\left(0, T ; L^{2}\left(\Gamma\left(x^{0}\right)\right)\right)}^{2} .
$$

At this time, we get a control

$$
\phi= \begin{cases}\psi & \text { on } \Sigma\left(x^{0}\right) \\ 0 & \text { on } \Sigma_{*}\left(x^{0}\right) .\end{cases}
$$


Acknowledgment. The first author is grateful for the Overseas Postgraduate Research Scholarship from the Australian Government, the University of Wollongong Postgraduate Research Award, and the Department of Mathematics Analysis Research Group Scholarship from the Faculty of Informatics at the University of Wollongong. The authors thank the anonymous referee for bringing the papers [1], [4], [5], [9] to their attention.

\section{REFERENCES}

[1] N. Burq, Contrôle de l'équation des plaques en présence d'obstacles strictement convexes, Mém. Soc. Math. France (N.S.) 55, 1-126 (1993)

[2] R. Dautray and J. L. Lions, Mathematical analysis and numerical methods for science and technology, Vol. 2, Functional and Variational Methods, Springer-Verlag, Berlin, 1992

[3] R. Dautray and J. L. Lions, Mathematical analysis and numerical methods for science and technology, Vol. 5, Evolution Problems I, Springer-Verlag, Berlin, 1992

[4] A. Haraux, Séries lacunaires et contrôle semi-interne des vibrations d'une plaque rectangulaire, J. Math. Pures et Appl. 68, 457-465 (1989)

[5] S. Jaffard, Contrôle interne exact des vibrations d'une plaque carrée, C. R. Acad. Sci. Paris 307, Série I, 759-762 (1988)

[6] V. Komornik, Exact controllability and stabilization: The multiplier method, John Wiley and Sons, Masson, Paris, 1994

[7] I. Lasiecka and R. Triggiani, Exact controllability of the Euler-Bernoulli equation with controls in the Dirichlet and Neumann boundary conditions: A nonconservative case, SIAM J. Control and Optimization 27, No. 2, 330-373 (1989)

[8] I. Lasiecka and R. Triggiani, Further results on exact controllability of the Euler-Bernoulli equation with controls on the Dirichlet and Neumann boundary conditions, Stabilization of Flexible Structures (Montpellier, 1989), Lecture Notes in Control and Information Sciences, 147, Springer-Verlag, 1990, pp. 226-234

[9] G. Lebeau, Contrôle de l'équation de Schrödinger, J. Math. Pures et Appl. 71, 267-291 (1992)

[10] J. L. Lions, Contrôlabilité exacte perturbations et stabilisation de systèmes distribués, Tome 1, Contrôlabilité Exacte, Masson, Paris, Milan, Barcelone, Mexico, 1988

[11] J. L. Lions, Exact controllability, stabilization and perturbations for distributed systems, SIAM Review 30, no. 1, 1-68 (1988)

[12] J. L. Lions and E. Magenes, Non-homogeneous boundary value problems and applications, Vols. I and II, Springer-Verlag, Berlin, Heidelberg, New York, 1972

[13] J. Simon, Compact sets in the space $L^{p}(0, T ; B)$, Annali di Matematica Pura ed Applicata (IV), Vol. CXLVI, 1987, pp. 65-96

[14] E. Zuazua, Exact boundary controllability for the semilinear wave equation, in Nonlinear Partial Differential Equations and their Applications, Collège de France Seminar, Vol. X (Paris, 1987-1988) 357-391, Pitman Research Notes in Mathematics Series 220, Longman Science and Technology, Harlow, 1991 (H. O. Fattorini) 